Revue des patrimoines

17 | 2011

Les patrimoines de l'enseignement supérieur

\title{
Les collections scientifiques de l'université Bordeaux 1. Sciences et Technologies
}

Jérôme de La Noë

\section{OpenEdition}

Journals

Édition électronique

URL : http://journals.openedition.org/insitu/1987

DOI : 10.4000/insitu. 1987

ISSN : 1630-7305

Éditeur

Ministère de la culture

Référence électronique

Jérôme de La Noë, « Les collections scientifiques de l'université Bordeaux 1. Sciences et Technologies », In Situ [En ligne], 17 | 2011, mis en ligne le 14 février 2012, consulté le 30 avril 2019. URL : http:// journals.openedition.org/insitu/1987 ; DOI : 10.4000/insitu. 1987

Ce document a été généré automatiquement le 30 avril 2019

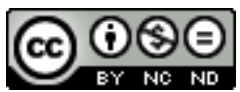

In Situ Revues des patrimoines est mis à disposition selon les termes de la licence Creative Commons Attribution - Pas d'Utilisation Commerciale - Pas de Modification 4.0 International. 


\title{
Les collections scientifiques de l'université Bordeaux 1. Sciences et Technologies
}

\author{
Jérôme de La Noë
}

\section{Introduction'}

1 De nombreuses institutions telles que fondations, musées, bibliothèques, archives ou sociétés savantes ont été conduites, de par leur constitution ou leur histoire, à conserver des collections d'objets très divers, d'œuvres d'art, de manuscrits et d'ouvrages anciens, etc. Ces collections ont été rassemblées par des hommes qui ont pris part à la vie de l'institution ou ont souhaité remettre les collections qu'ils ont rassemblées à l'institution de leur choix. De nombreuses universités se trouvent être détentrices de collections, en général constituées par des professeurs ou des personnels universitaires. C'est ainsi que l'université Bordeaux 1, Sciences et Technologies, dispose de neuf grandes collections. Comme nous le verrons par la suite, celles-ci sont dues à des hommes, en majorité universitaires, mais pas uniquement car plusieurs amateurs ont également contribué. Quelle est alors la motivation de ces collecteurs ou collectionneurs: la passion? La raison? Le raisonnement scientifique? La passion de l'enseignement universitaire? Différentes motivations peuvent se mêler dans différentes proportions pour chaque collectionneur.

2 Les collections de l'université Bordeaux 1 ont des origines très diverses. Certaines ont été constituées dès le milieu du XIX ${ }^{e}$ siècle. Au cours du $\mathrm{XX}^{\mathrm{e}}$ siècle, certaines continuent d'être complétées, d'autres non. Elles n'en deviennent pas pour autant obsolètes, mais témoignent d'un riche patrimoine scientifique. D'autres enfin, telle la carothèque océanographique, sont en cours de constitution. Elles sont, pour certaines, constituées de milliers de spécimens de référence rassemblés au cours des décennies mais correspondant à des périodes géologiques très anciennes, à des sites paléontologiques aquitains ou de différents continents, des pièces inestimables, des instruments 
scientifiques originaux, mais encore des papillons, des oiseaux, de multiples insectes... À côté des muséums, les universités ont une responsabilité très importante dans la conservation des collections qu'elles ont constituées. Ces collections sont actuellement utilisées pour l'enseignement et/ou la recherche, ou servent de collections d'étude. Quelle que soit leur utilisation, elles constituent un patrimoine scientifique d'un intérêt majeur et donc des collections patrimoniales dont la conservation devrait être un impératif pour leurs tutelles. De plus, les collections, en rassemblant des objets fabriqués à différentes périodes, permettent de mieux appréhender l'évolution des méthodes utilisées et des techniques inventées par l'homme au cours des âges, des siècles et des dernières décennies. Les collections participent donc à l'histoire des sciences par l'étude des méthodologies mises en œuvre par l'homme.

Les raisons pour lesquelles cette étude est présentée sont diverses. D'une part, par le travail incessant accompli par les responsables de collections, les instances universitaires prennent peu à peu conscience de la valeur et de la richesse des collections de l'université. En parallèle, le recensement méticuleux des collections progresse différemment selon leur nature et les forces qui peuvent s'y investir. Les responsables portent également le souci du devenir de ces collections au sein de l'université, car selon leur nature, les problèmes de conservation, de protection et d'inventaire sont différents. Cette prise de conscience a été rendue possible depuis plusieurs années par différentes actions menées au sein de l'université, comme la publication d'une plaquette ${ }^{2}$ résumant les caractéristiques principales de chaque collection, une vidéo ${ }^{3}$ illustrant certains aspects de l'état actuel de la conservation, des ouvertures au public lors de journées "portes ouvertes" sur le campus. Enfin une exposition est en cours de préparation en collaboration avec le Centre de Culture Scientifique, Technique et Industrielle Cap Sciences avec la participation du Muséum d'Histoire naturelle de Bordeaux, afin de présenter de façon pédagogique les collectionneurs et leurs motivations, ainsi que les fonctions pour lesquelles les collections sont actuellement utilisées: enseignement, recherche, patrimoine, histoire des sciences...

On peut dès à présent passer en revue les différentes collections de l'université Bordeaux 1.

\section{Les collections de Préhistoire}

5 Les collections de préhistoire de l'UMR 5199 «De la Préhistoire à l'Actuel: Culture, Environnement et Anthropologie ${ }^{4} »$ (PACEA) sont constituées d'outils en pierre taillée, d'ossements humains et animaux, de céramiques et d'échantillons de sédiments. La majorité du matériel est paléolithique et concerne surtout les parties les plus récentes de cette période, paléolithique moyen et supérieur. S'y ajoutent quelques objets d'autres époques, y compris historiques. Les séries proviennent essentiellement du sud-ouest de la France, mais quelques-unes sont issues d'autres régions, même de contrées plus lointaines : Afrique, Australie, Europe de l'Est.

6 Les « séries anciennes » sont composées de petits ensembles juxtaposés. Pour chaque site archéologique, on ne dispose que de quelques objets qui, pour la plupart, ont été sélectionnés selon des critères esthétiques. Ces séries sont le reflet des techniques de fouille de l'époque : on ne ramassait pas l'intégralité du matériel. Pour les outils en silex, on collectait surtout les outils finis (bifaces, racloirs, grattoirs, haches polies) et pour les restes d'animaux, les os entiers ou les extrémités articulaires étaient privilégiés. Au 
détour de certains tiroirs, les séries anciennes, parfois seuls témoins pour des sites aujourd'hui disparus, sont aussi une "mémoire de l'histoire des sciences préhistoriques et de leur évolution ".

7 Au fil des années, les fouilles sont devenues de plus en plus minutieuses et la collecte de plus en plus exhaustive. Aujourd'hui, les sédiments sont tamisés, ce qui permet de récolter du matériel de très petite taille, tels les micro-éclats de silex ou des ossements de rongeurs. Pour cette raison, les « séries récentes » sont très importantes et exposent une plus grande variété des matériaux (fig. $\left.\mathbf{n}^{\circ} \mathbf{1}\right)$.

Figure 1

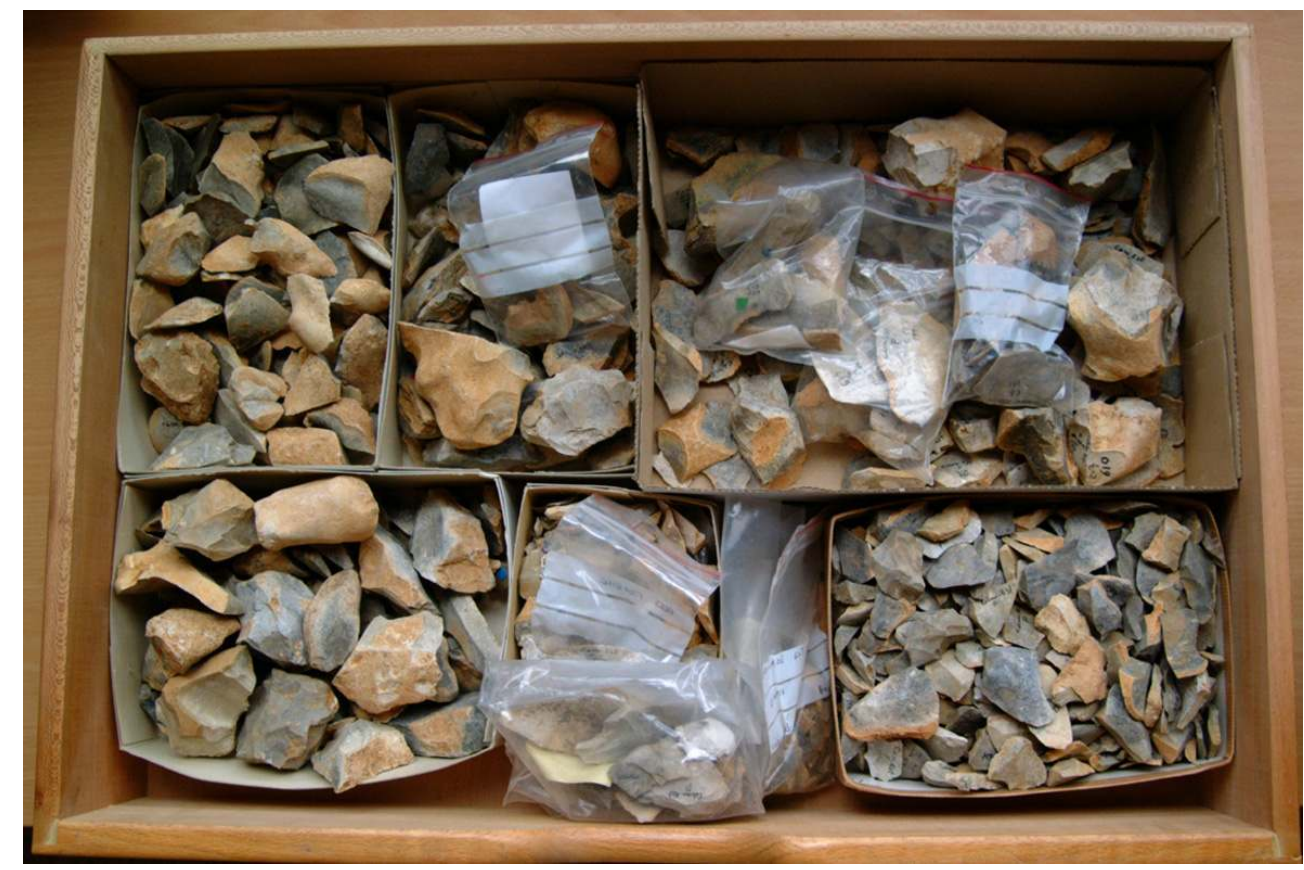

Série lithique du site de Caminade (Dordogne). Collections de préhistoire de l'UMR 5199, IPGQ/PACEA. Phot. Olivier Got. @ Service Communication, Université Bordeaux 1.

Ces séries de préhistoire ont conservé la « mémoire des hommes » qui les ont constituées. En premier lieu, le professeur Georges Malvesin-Fabre (1893-1956), fondateur de la chaire de préhistoire à la faculté des Sciences de Bordeaux, a réuni des collections pour les utiliser dans le cadre de son enseignement. Malheureusement, il ne les utilise pas, puisqu'il meurt en 1956, la veille de l'ouverture du poste de professeur. Certaines de ces séries servent encore pour les enseignements du master Anthropologie biologique et Préhistoire. Les collections de G. Malvesin-Fabre ont été obtenues par dons (en particulier ceux de la faculté de Médecine de Bordeaux) ou acquisitions (fig. $\mathbf{n}^{\circ} \mathbf{2}$ ). 


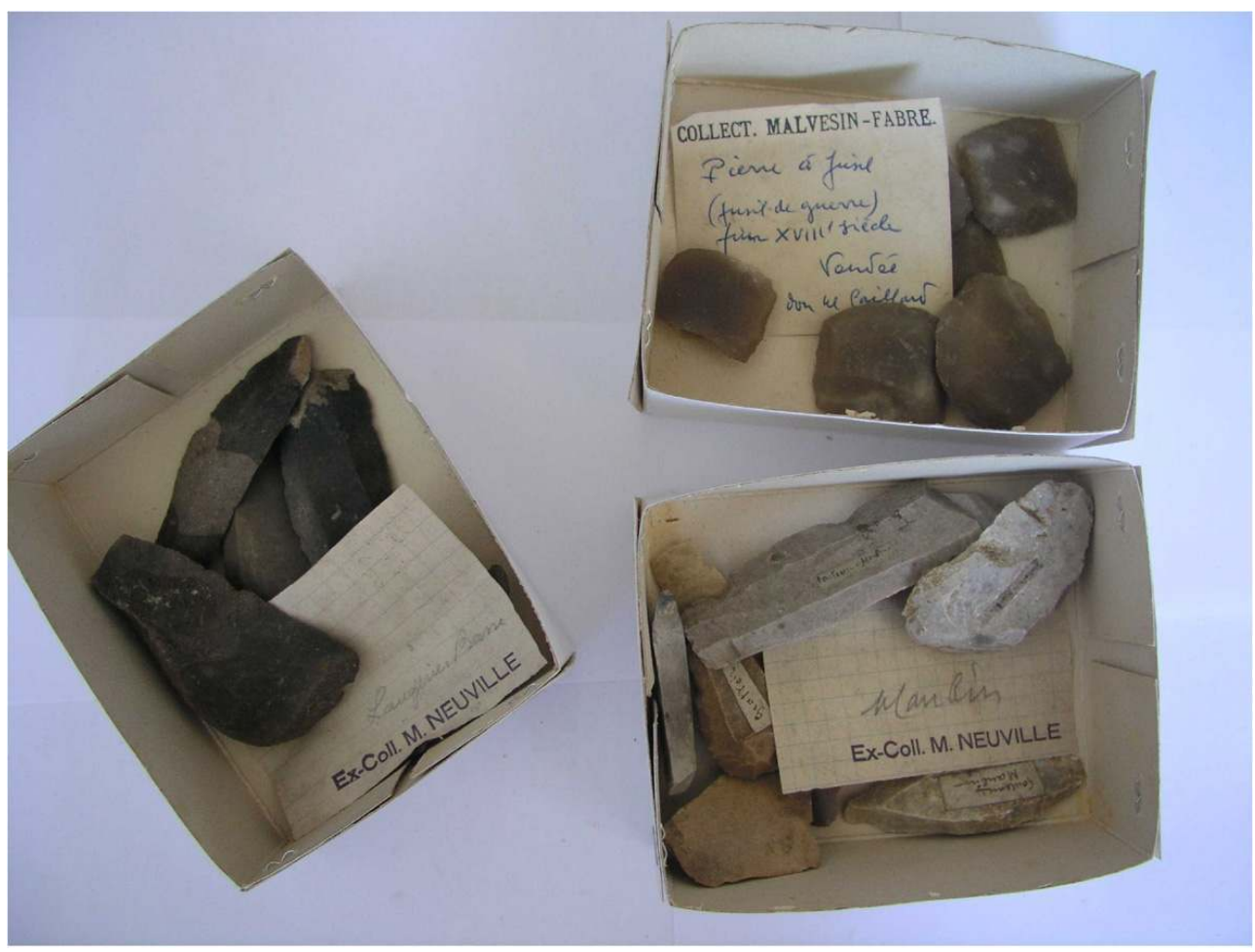

Quelques exemplaires de pièces des collections rassemblées par Georges Malvesin-Fabre et Marcel Neuville. Collections de préhistoire de I'UMR 5199, IPGQ/PACEA.

Phot. Olivier Got. ( ) Service Communication, Université Bordeaux 1.

Pour les acquisitions, l'un des ensembles importants est la « collection Neuville». Marcel Neuville vivait à Bordeaux fin $\mathrm{XIX}^{\mathrm{e}}$ - début $\mathrm{XX}^{\mathrm{e}}$ siècle. Il était ébéniste et naturaliste amateur. L'histoire de M. Neuville est celle de nombreux collectionneurs de cette époque. Il collectionnait dans plusieurs domaines, en particulier la préhistoire et la paléontologie. Il n'existe pas d'articles écrits par M. Neuville dans le bulletin de la Société archéologique de Bordeaux, seulement quelques contributions en paléontologie dans celui de la Société linnéenne : c'était avant tout un collectionneur. Une partie seulement de ses collections a été achetée par la faculté des Sciences de Bordeaux, dont un important ensemble de fossiles aquitains (cf. infra). En avance sur son temps, M. Neuville pratiquait l'expérimentation, il avait fabriqué des reconstitutions d'armes de jet en réunissant ses connaissances en préhistoire et ses talents d'ébéniste.

La présence dans les collections de l'Institut de Préhistoire et de Géologie du Quaternaire, d'une petite série d'éolithes découverte par Jean-Baptiste Rames (1832-1894) en 1877 dans les niveaux tertiaires de Puy-Courny (Cantal) est un autre exemple. Ces objets apportent le témoignage des débuts de la construction des sciences préhistoriques. Ils ont alimenté un débat sur l'origine de l'humanité et sur l'ancienneté de l'homme. Les éolithes de PuyCourny sont des blocs de silex, sur lesquels on voit des enlèvements d'éclats plus ou moins grands et qui ont été retrouvés dans des niveaux déposés par un cours d'eau et datés du Miocène récent. Invitées par J.-B. Rames, des sommités scientifiques de l'époque vont visiter le site pour débattre « in situ » de l'origine de ces éolithes : ont-ils été taillés par l'homme? 
11 L'absence d'ossements humains associés à ces industries du Tertiaire et les positionnements stratigraphiques souvent incertains des vestiges laissent planer un doute sur la validité de ces découvertes. Les études et expérimentations réalisées au début $\mathrm{du} \mathrm{XX}^{\mathrm{e}}$ siècle ne firent qu'amplifier ce doute et vinrent prouver que des processus naturels, comme la pression du sédiment, les chocs thermiques, les éboulements ou le transport par les rivières, pouvaient produire des outils rudimentaires semblables à ceux qui sont découverts dans les niveaux tertiaires. Dès le début du XX $\mathrm{X}^{\mathrm{e}}$ siècle, l'idée d'une industrie éolithique et d'un homme tertiaire était quasiment enterrée. La tentative d'accéder à nos origines en s'appuyant sur les éolithes s'est donc soldée par un échec.

Actuellement, ces collections sont sous la responsabilité de Dominique Armand et Éric Pubert, de l'UMR 5199 PACEA.

\section{Les collections d'Anthropologie}

La constitution des collections anthropologiques a débuté à l'université Bordeaux 1 avec la création, au début des années 1970, du laboratoire d'Anthropologie par le Dr Raymond Riquet (1914-1983), médecin qui a occupé le premier poste de professeur d'Anthropologie à l'université ${ }^{5}$. À cette époque, le travail du paléoanthropologue ne concernait que des analyses en laboratoire. La fouille et le prélèvement des vestiges anthropologiques étaient réalisés par les archéologues. Seules les découvertes de squelettes anciens du Paléolithique amenaient les spécialistes sur les sites. Les premières collections conservées au sein de l'université concernaient le Néolithique français, avec quelques sites étrangers, d'Afrique du Nord principalement.

Au début des années 1980, le squelette n'est plus considéré comme secondaire, mais il est devenu l'élément central de la tombe. La position archéologique du squelette devient alors primordiale pour comprendre le fonctionnement de la sépulture. Cette approche a été particulièrement développée au sein du laboratoire PACEA et elle est rapidement devenue l'un de ses axes fondateurs. Plusieurs dizaines d'étudiants et d'archéologues ont ainsi été formés à cette approche anthropologique des sépultures. L'essor de l'archéologie préventive, ainsi que la reconnaissance de cette discipline, dite anthropologie de terrain, ont contribué à l'exhumation d'innombrables squelettes. Grâce aux relations que le laboratoire a développées et entretenues, de nombreux étudiants sont devenus des acteurs de cette archéologie de sauvetage et sont à l'origine de l'arrivée de plusieurs centaines de collections actuellement conservées au Centre anthropologique de Pessac, Gironde $^{6}$. Il s'agit d'une structure tout à fait unique en France et dont la réalisation a bénéficié de la collaboration de la ville de Pessac, de la Direction régionale des Affaires culturelles (DRAC) et de l'université. Elle contient actuellement près de 300 séries ostéologiques, d'origines géographiques, chronologiques et culturelles différentes.

partie de ces séries constitue une ostéothèque, c'est-à-dire un ensemble de séries de référence. Toute étude paléoanthropologique impose des comparaisons qui font appel à des données différentes selon les thématiques concernées. Les recherches menées portent sur différents thèmes.

Le premier thème correspond à "Origine et évolution d'Homo sapiens » : l'interprétation morphologique et fonctionnelle des pièces fossiles paléolithiques ne peut être abordée qu'en fonction de la variabilité observée dans les populations relativement récentes. Or, les collections de squelettes actuels sont extrêmement dispersées; la provenance des 
sujets qui les composent est, par ailleurs, mal connue. Il est donc indispensable de pouvoir disposer de collections nombreuses et bien documentées; le matériel issu de fouilles menées dans des conditions d'observations satisfaisantes constitue une référence de choix. Par ailleurs, l'approche de la variabilité peut ainsi se fonder sur des séries d'âges divers, en fait à partir de l'apparition des gisements regroupant des effectifs importants, c'est-à-dire depuis le Néolithique. Ces variations peuvent concerner les dimensions, mais plus encore la morphologie détaillée des structures osseuses (insertions musculaires et ligamentaires, orifices, surfaces articulaires...).

Un second thème concerne les "Analyses biologiques des séries d'époques protohistorique et historique " : l'analyse aura deux orientations distinctes et complémentaires. La première réside dans une approche biologique de la population concernée, l'échantillon est alors considéré comme représentatif de celle-ci, ou biaisé d'une manière telle qu'il est possible de corriger les anomalies. L'autre voie interprétative concernera la restitution des règles qui ont présidé au recrutement de l'ensemble funéraire et à la distribution des sujets au sein de celui-ci.

Actuellement, des études de populations archéologiques sont effectuées à Pessac. Celles-ci se font, soit dans le cadre de travaux universitaires, soit dans celui de contrats d'étude sur des séries provenant de fouilles préventives. Ces travaux ne font pas directement appel à l'ostéothèque, mais viennent l'alimenter tant en matériel qu'en résultats. Les contrats d'études primaires ne permettent pas d'exploiter certains aspects de recherche, mais ils révèlent, bien souvent, certaines particularités qui seront par la suite abordées dans le cadre d'études spécifiques.

Figure 3

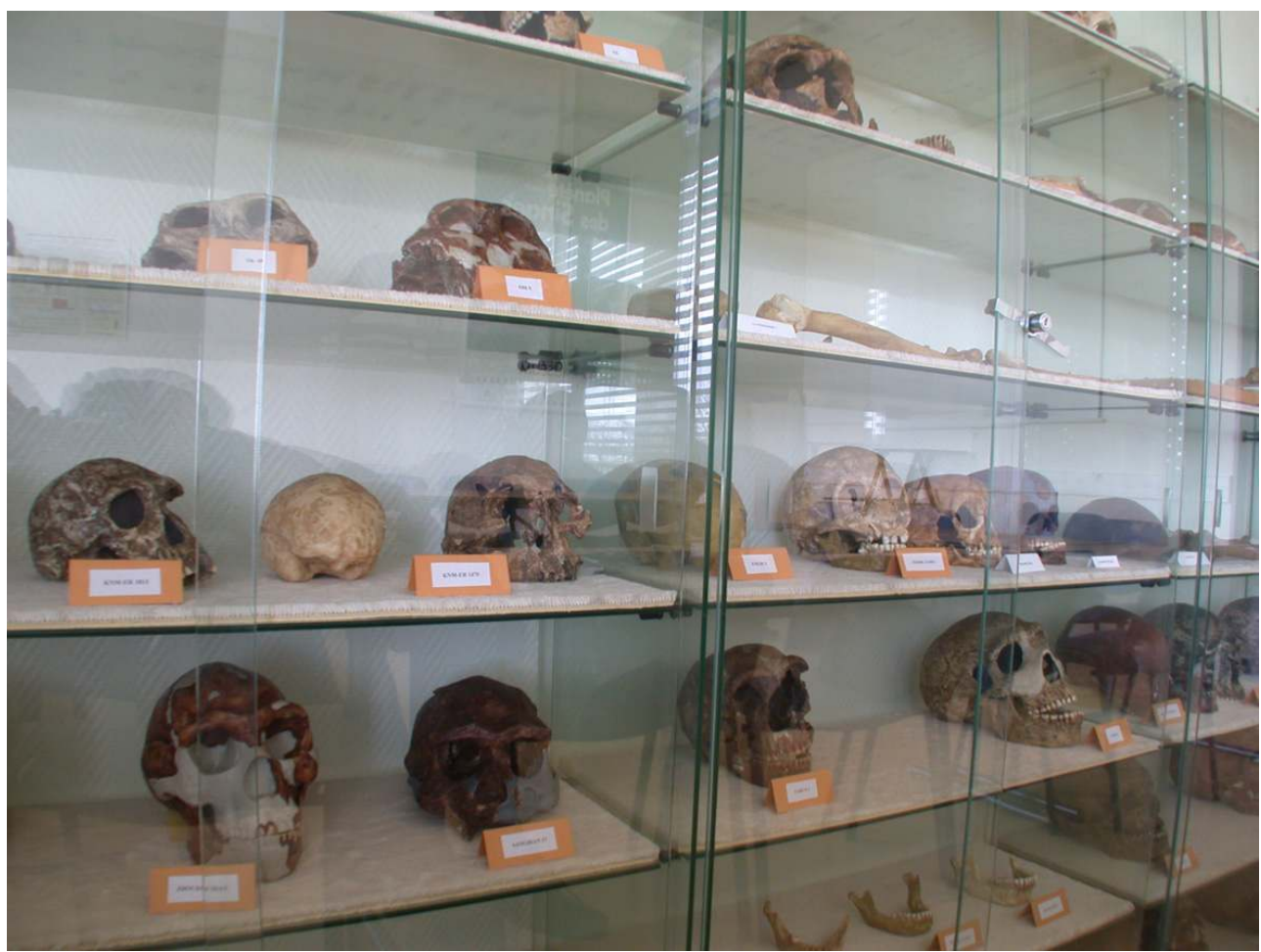

Collection de moulages, témoins de l'évolution humaine. Collections de l'équipe « Anthropologie des Populations Passées et Présentes ».

Phot. Maryelle Bessou. (c) A3P, Université Bordeaux 1. 
19 À ces séries ostéologiques humaines vient s'ajouter une importante «collection de moulages de pièces fossiles humaines " (crânien et infra-crânien) de près de 1700 pièces (fig. $\mathbf{n}^{\circ 3}$ ). Elle fut constituée dès les années 1980 par des moulages réalisés au sein même $\mathrm{du}$ laboratoire d'anthropologie alors que les fragments originaux étaient encore assez aisément mis à disposition des anthropologues quel que soit leur site (étranger ou français) d'origine. Ensuite, la réglementation internationale et l'inaccessibilité des fossiles originaux en raison de problèmes de conservation ont conduit à acheter et échanger des moulages avec différents instituts de recherche et les musées en France et à l'étranger. Ces moulages représentent un patrimoine scientifique à part entière pour la communauté des chercheurs et enseignants. La collection dispose ainsi de matériels fossiles provenant d'horizons géographiques, chronologiques et culturels très divers retraçant l'évolution humaine. Leur présence en un seul lieu permet leur manipulation et leur étude sans avoir à se déplacer à travers le monde. C'est ce qui a motivé la constitution de cette riche collection à son origine.

Peu à peu, elle s'est enrichie également de moulages des premières sépultures, documents iconographiques uniques témoignant ainsi de l'état de la tombe au moment précis de sa mise au jour. Comme ces sépultures ont été dégagées et les vestiges prélevés, on comprend alors aisément la valeur même de ces moulages (fig. $\left.\mathbf{n}^{\circ} \mathbf{4}\right)$.

Figure 4

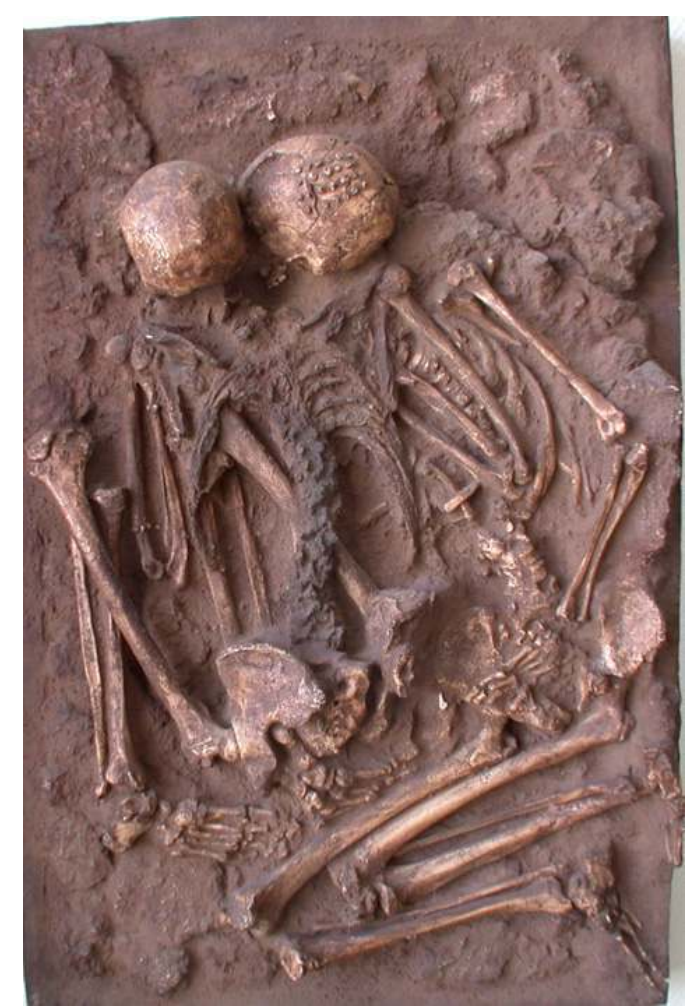

Moulage de la « Grotte des Enfants », Site de Baoussé-Roussé, Grimaldi, Italie. Aurignacien, Paléolithique supérieur, - 25000 ans. Les deux sujets, l'adolescent à gauche, la femme à droite, ont été ensevelis en même temps. Le dépôt est accompagné d'objets de parure. Collections de l'équipe "Anthropologie des Populations Passées et Présentes ».

Phot. Maryelle Bessou. (C) A3P, Université Bordeaux 1.

Les collections permettent de mener des travaux de recherche spécifiques touchant le domaine de l'évolution, au travers d'études comparatives notamment, ainsi que dans le 
cadre de mémoires universitaires. Des études morphologiques sont menées, et désormais l'imagerie 3D élargit nos potentialités d'étude plus approfondie de ces moulages de pièces fossiles.

Actuellement, ces collections sont sous la responsabilité de Patrice Courtaud et Maryelle Bessou, de l'UMR PACEA.

\section{Les collections de Géologie}

La carothèque-lithothèque historique a été créée par les personnels de l'Institut de Géologie du Bassin d'Aquitaine dans les années 1960 et elle est actuellement gérée par l'UfR des Sciences de la Terre et de la Mer. Cette collection est conservée dans les communs du château Bonnefont, l'un des bâtiments de l'université. Elle regroupe un ensemble complet d'échantillons géologiques représentatifs de tout le Bassin aquitain, prélevés soit en surface (affleurements), soit en profondeur (forages). 35000 échantillons de terrain et des carottes issues de 1600 forages réalisés principalement de 1950 à nos jours sont classés et répertoriés. Cet ensemble actuellement sauvegardé constitue une collection unique en Aquitaine et la seule carothèque existante pour ce bassin (fig. $\mathbf{n}^{\circ} \mathbf{5}$ ).

Figure 5

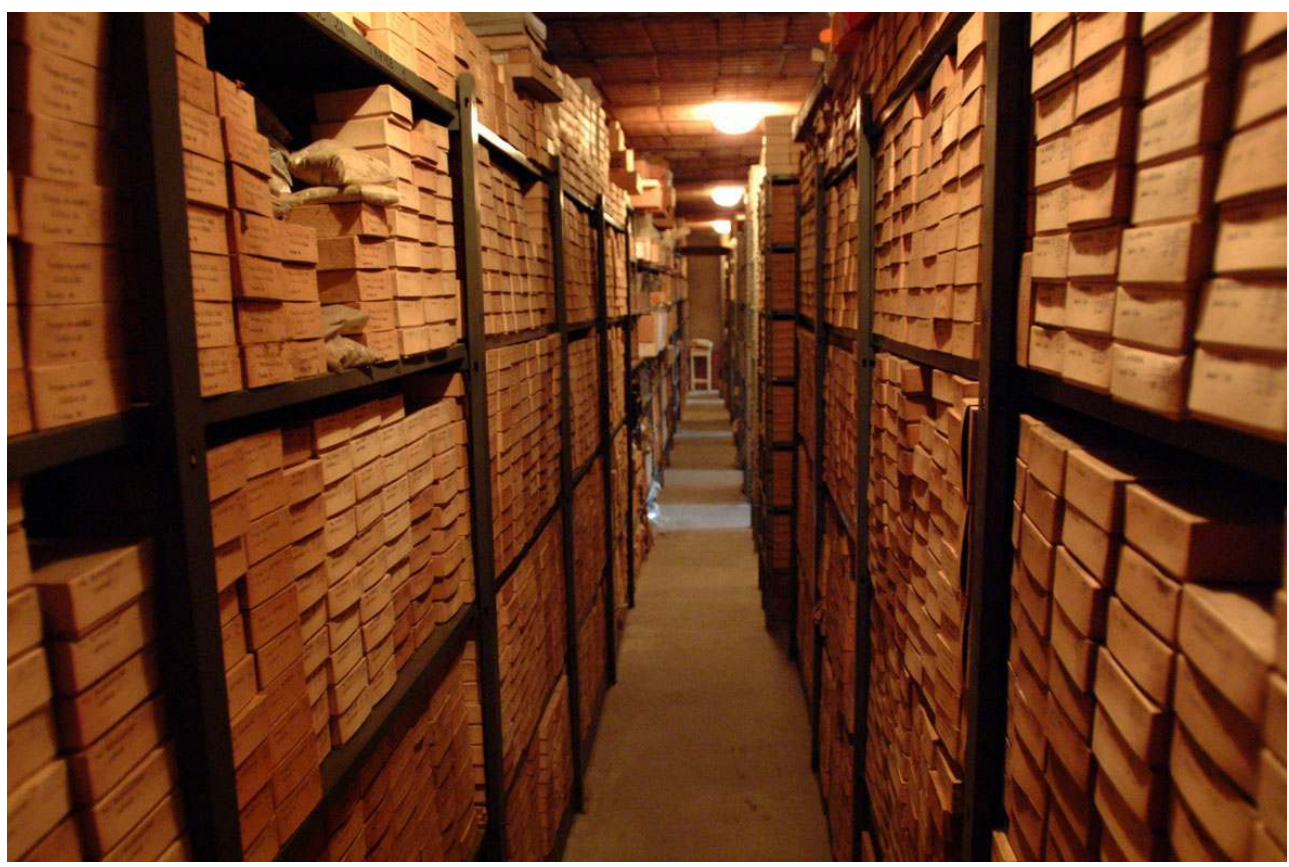

Vue de la carothèque-lithothèque de l'Université Bordeaux 1 ; les petites boîtes cartonnées contiennent les résidus bruts de forages recueillis selon les profondeurs. Collections de l'UFR des Sciences de la Terre et de la Mer.

Phot. Bruno Cahuzac. (c) Université Bordeaux 1.

La disparition progressive et généralisée des affleurements, des carrières et des marnières en Aquitaine montre tout l'intérêt de la conservation des échantillons prélevés, qui constitue une véritable «mémoire patrimoniale» du sous-sol. $97 \%$ des forages sont issus de travaux de recherches en eau et de génie civil, et $3 \%$ proviennent de recherches pétrolières. La série complète de forages constitue un cas d'application de la recherche à des nécessités économiques quotidiennes, ces forages constituant des outils 
irremplaçables pour la connaissance géologique. Ainsi, les données recueillies sont utiles pour les recherches en eau dont les besoins augmentent régulièrement pour l'irrigation et la consommation humaine. Le Bassin aquitain comporte plusieurs nappes aquifères déjà exploitées et d'autres susceptibles d'être potentiellement utilisées. D'autre part, les études et les ouvrages effectués sur le territoire de la Communauté urbaine de Bordeaux ont été nombreux. Les données de sondages seront toujours d'actualité en ce qui concerne les constructions, déjà réalisées ou à venir (parkings souterrains, fondations, ponts... et autres ouvrages de génie civil), ou les questions d'environnement (périmètres de protection, cimetières, sources, pollution de nappes ou de rivières). En cas d'expertise du sous-sol en Aquitaine, le besoin d'avoir des références scientifiques précises des échantillons (datations, épaisseurs, description des niveaux), et d'examiner des sédiments disponibles en carothèque, s'avère nécessaire (fig. $\left.\mathbf{n}^{\mathbf{0}} \mathbf{6}\right)$. Enfin, d'autres forages plus profonds ont servi à la recherche d'hydrocarbures.

Figure 6

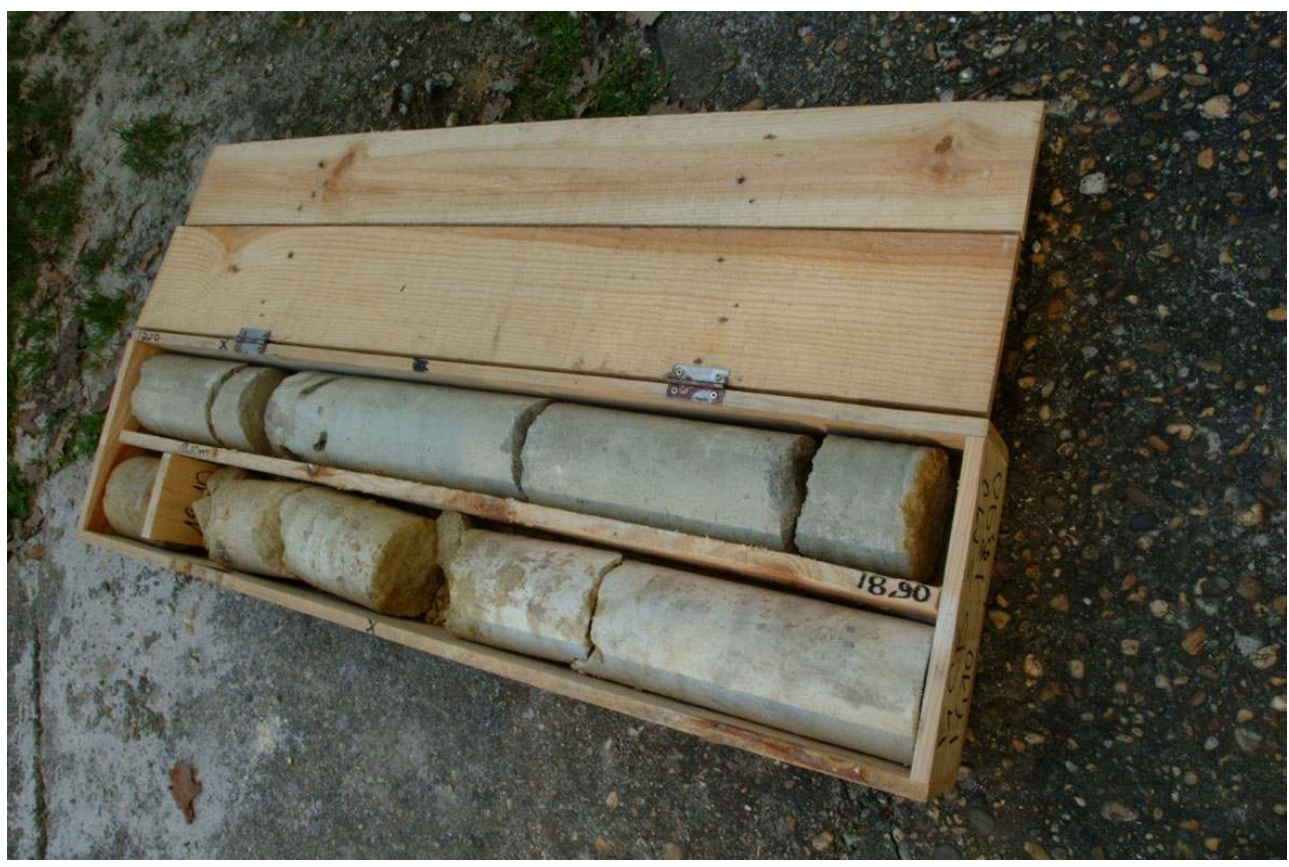

Des tronçons de carotte prélevés dans des sédiments durs du sous-sol girondin, et datant de l'ère tertiaire (Oligocène). Collections de I'UFR des Sciences de la Terre et de la Mer.

Phot. Bruno Cahuzac. (C) Université Bordeaux 1.

Les échantillons des 1600 forages sont associés à un dossier comportant des renseignements sur la localisation, le cadre géologique, les coupes, les descriptions lithologiques, la stratigraphie et souvent sur les débits en eau par jour ; figurent aussi des enregistrements de diagraphies (électriques et nucléaires). Les données de base ont récemment fait l'objet d'un inventaire informatisé. Ces forages sont toujours utilisés de façon pérenne en recherche fondamentale. Il apparait, au vu des nombreuses thèses et publications, que ce matériel sédimentaire est devenu une référence nationale, voire internationale, en particulier pour les fossiles qu'il contient et qui servent de base de comparaison et d'outil de corrélation stratigraphique de grande importance (macrofaune, microfaune marines). Des études micropaléontologiques détaillées (Foraminifères, 
Ostracodes, Bryozoaires...) sont encore aujourd'hui réalisées sur ces forages. Ceux-ci sont également utilisés pour l'établissement et/ou la révision des cartes géologiques.

De nouveaux outils et techniques d'investigation permettent des recherches sur le matériel de cette carothèque-lithothèque. Actuellement, sont menées des études de lithostratigraphie, de biostratigraphie et d'analyses séquentielles des dépôts sédimentaires de tous types, littoraux, profonds, lagunaires et continentaux, qui contribuent à la paléogéographie régionale. Des investigations sur d'autres groupes fauniques sont aussi envisageables à l'avenir.

La carothèque-lithothèque fait partie du patrimoine scientifique de l'université. Il s'agit d'un ensemble répertorié dont la valeur est liée à l'inventaire (collection d'étude) et à l'aspect recherche (objets décrits et observés). La conservation d'un tel ensemble (qui ne pourra plus être renouvelé) constitue une mission de service public. C'est aussi une collection historique et régionale liée aux laboratoires universitaires successifs qui, depuis les années 1950, ont collecté les échantillons de forage et de terrain. Ce patrimoine historique remonte aux premiers temps de la faculté des Sciences de Bordeaux, au milieu du XIX ${ }^{e}$ siècle ${ }^{7}$. Déjà en 1868, Victor Raulin (1815-1905) (fig. $\mathbf{n}^{\circ} 7$ ) avait reconnu l'intérêt fondamental des forages et publié des coupes détaillées qui font encore référence ${ }^{8}$. Les échantillons conservés aujourd'hui intègrent notamment des prélèvements de terrain et des fossiles recueillis par des universitaires bordelais des $\mathrm{XIX}^{\mathrm{e}}$ et $\mathrm{XX}^{\mathrm{e}}$ siècles, comme les professeurs Victor Raulin, Hyacinthe de Collegno (1794-1856), Emmanuel Fallot (1857-1929), Fernand Daguin (1889-1948) (fig. nº $^{\circ}$ ), ainsi que L. Reyt (préparateur de géologie à la faculté dans les années 1890), puis André Magne, Michel Vigneaux et Jacques Alvinerie. 
Figure 7

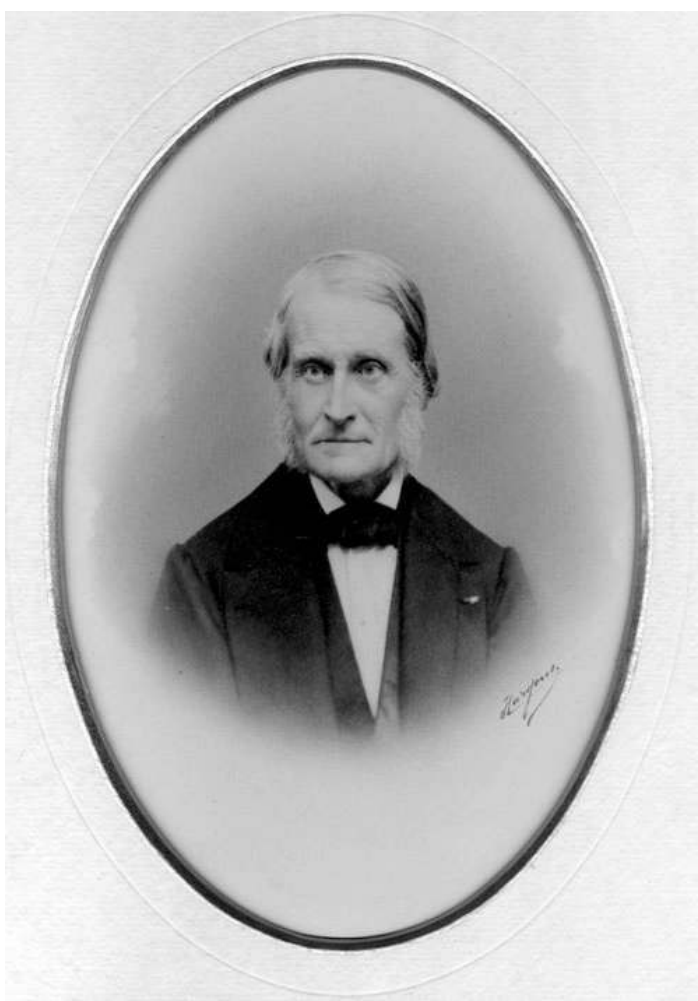

Portrait de Victor Raulin (1815-1905), professeur de géologie à la Faculté des Sciences de Bordeaux de 1846 à 1885.

Phot. P. Rocher. (c) P. Rocher. 


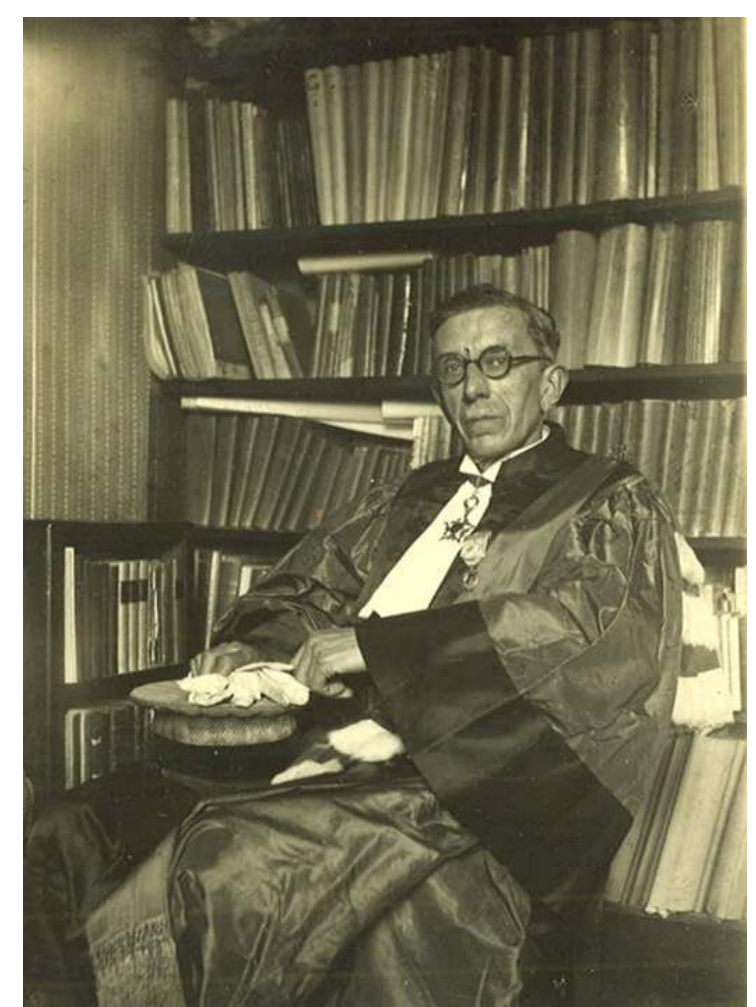

Portrait de Fernand Daguin (1889-1948), professeur à la Faculté des Sciences de Bordeaux de 1929 à 1948.

Phot. Bruno Cahuzac. (C) Université Bordeaux 1.

Enfin, cette carothèque est aujourd'hui la seule collection française, complète et représentative des séries géologiques du bassin aquitain, plus grand bassin sédimentaire français. L'université Bordeaux 1 s'honore de conserver un tel patrimoine scientifique, rassemblé sur le campus. Sur le plan national et international, la lithothèque contient des échantillons illustrant les étages géologiques qui ont été historiquement décrits en Aquitaine, et appartenant souvent aux coupes types (les stratotypes). Ainsi conserve-t-on des prélèvements des étages aquitanien, burdigalien, biarritzien, sallomacien, campanien, santonien, conacien, aturien. De tels sédiments, avec leurs fossiles associés, sont importants pour les recherches actuelles sur la connaissance et la validation de ces étages, et l'étude des marqueurs stratigraphiques qu'ils contiennent.

Cette description serait incomplète si on ne citait pas de beaux échantillons de cristaux appartenant à toutes les familles minéralogiques. Cette collection a été acquise, il y a une vingtaine d'années, pour son intérêt à la fois pédagogique, scientifique et esthétique dans l'enseignement. On peut y observer de nombreux types de minéraux présentés selon leur classe, ainsi que divers minerais.

Actuellement, ces collections sont sous la responsabilité de Bruno Cahuzac, de l'UFR des Sciences de la Terre et de la Mer. 


\section{Les collections de Minéralogie}

31 Une convention passée en septembre 2003 entre l'université Bordeaux 1 et la mairie de Bordeaux a permis de mettre en dépôt au Muséum d'Histoire naturelle la collection de minéraux de l'ancien laboratoire de Cristallographie de cette université. Cette collaboration fut le résultat de la démarche de Marc Alléaume, membre de ce laboratoire, qui souhaitait que la collection puisse être mise à disposition d'un public plus large dans le cadre du musée. Le transfert des douze meubles de vingt tiroirs chacun a été réalisé début octobre 2003 dans les réserves du Muséum, après couverture photographique des 240 tiroirs par l'équipe du Muséum (fig. $\mathbf{n}^{\circ} \mathbf{9}$ ). La collection est à présent mise à la disposition des chercheurs et des étudiants sur simple demande. Elle est aussi présentée au public lors des visites des réserves qui sont organisées chaque année à l'occasion des Journées nationales du Patrimoine.

Figure 9

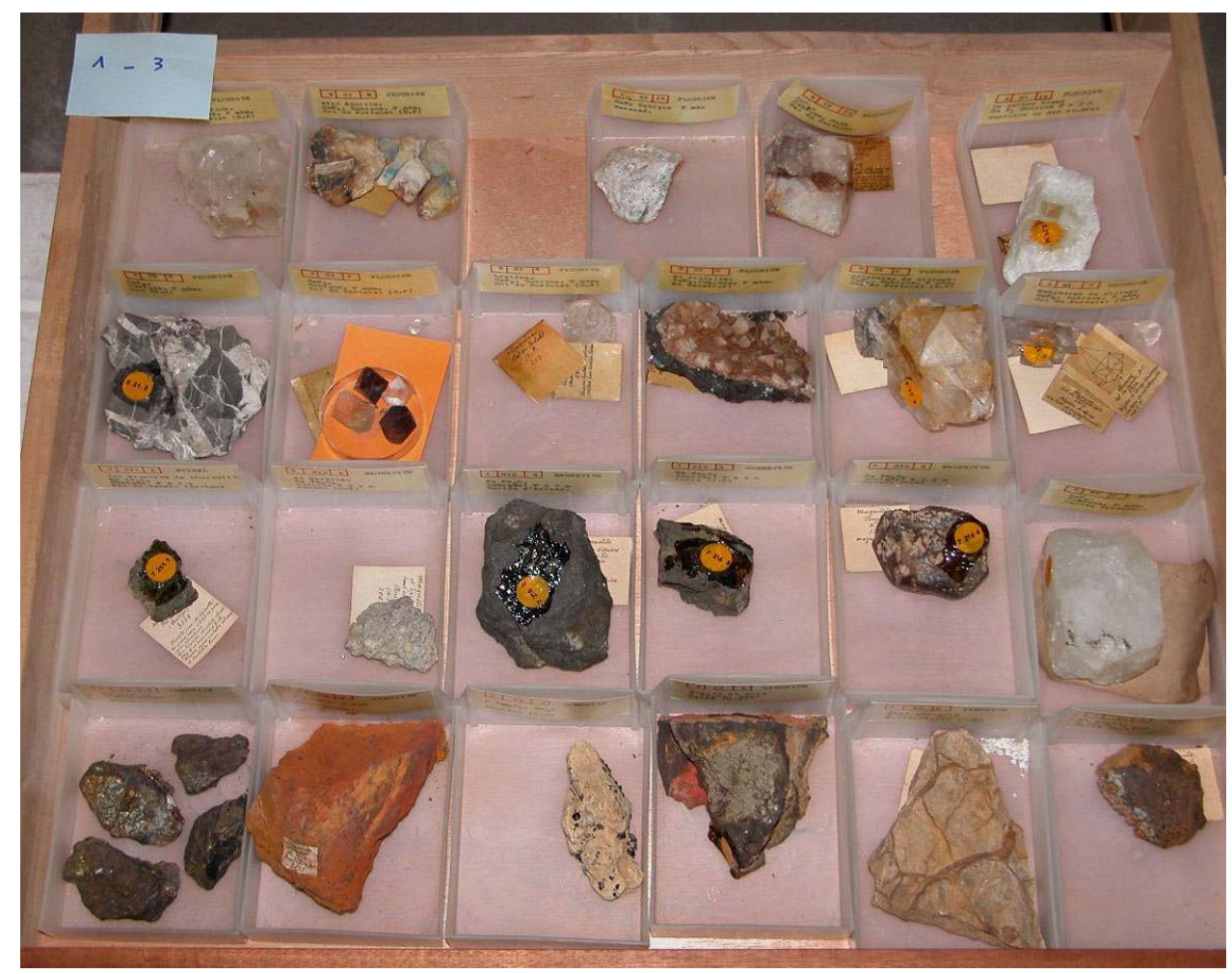

Un tiroir de la collection de minéralogie Robert Gay : échantillons des Pyrénées collectés par JeanLouis de Bonnal.

Phot. Muséum d'Histoire naturelle de Bordeaux. (c) Muséum d'Histoire naturelle de Bordeaux.

La collection est riche de plus de 5000 échantillons répertoriés. Elle comporte deux sections principales: d'une part, une collection de minéraux des Pyrénées, rassemblée par un amateur averti, E. Bonnel, puis achetée par le laboratoire en 1960. Avec 1000 entrées au fichier, elle n'a sans doute pas d'équivalent en France. D'autre part, une collection générale, qui provient du monde entier, compte 4000 entrées. Elle résulte de la fusion des collections initiales du laboratoire de Cristallographie et de la partie étrangère de la collection Bonnel que ce dernier avait constituée par échanges. 
33 À l'occasion du dépôt, il a été convenu que cette collection porterait le nom de "collection Robert Gay» en hommage à celui qui fut le principal artisan de sa constitution. Ainsi, le 10 mars 2004, une réception officielle a permis de réunir au Muséum les représentants de l'université et de la mairie de Bordeaux, les chercheurs de l'ancien laboratoire ainsi que de nombreuses personnalités scientifiques impliquées dans la recherche en cristallographie et minéralogie. La séance était animée par une conférence d'Hubert Curien (1924-2005), cristallographe, ancien ministre de la Recherche et fondateur de la Fête de la Science. Le récolement et l'informatisation de l'inventaire de cette collection ont été entrepris par l'équipe du Muséum en 2008 et se poursuivent en 2009. Un peu plus de la moitié de la collection a déjà été saisie. Le Muséum fait aujourd'hui l'objet d'un projet de rénovation et d'extension dont l'ouverture est prévue en 2012 ; une sélection d'échantillons de la collection permettra d'illustrer la partie du futur parcours permanent consacrée à la minéralogie.

Actuellement, ces collections sont sous la responsabilité de Nathalie Mémoire, conservateur.

\section{Les collections de Paléontologie et Micropaléontologie}

L'université Bordeaux 1 possède de très riches collections paléontologiques, qui sont de grand intérêt scientifique. Plus de 100000 spécimens au total sont ainsi conservés. Ils sont principalement marins, avec quelques échantillons de fossiles terrestres ou d'eau douce.

Ces collections, soigneusement réunies par des scientifiques réputés, témoignent de l'histoire de l'université et de l'histoire des sciences en général. Plusieurs professeurs de la faculté des Sciences de Bordeaux, souvent précurseurs en paléontologie, ont légué à l'université le fruit d'une vie de récoltes et de fouilles. Citons Victor Raulin, Emmanuel Fallot, Fernand Daguin. Des amateurs éclairés ont aussi considérablement enrichi ces collections patrimoniales, comme le Dr Sylvestre de Grateloup, Marcel Neuville, Albert Peyrot, le Dr Louis Castex, le comte Gaëtan O'Gorman... Par exemple, deux meubles de la « collection Peyrot» (constituée ca 1900-1935) contiennent des mollusques, actuels et fossiles, conservés dans une série impressionnante de plus de 5000 boîtes d'allumettes, toutes étiquetées.

Une des collections les plus précieuses et les plus anciennes de macrofossiles (1815-1840) est celle du Dr Jean-Pierre Sylvestre de Grateloup ${ }^{10}$ (1782-1861) (fig. $\mathbf{n}^{\circ}{ }^{\circ}$ ) (fig. $\left.\mathbf{n}^{\circ}{ }^{111}\right)$. Ce dacquois fut un naturaliste pluridisciplinaire (géologie, paléontologie, botanique, zoologie, médecine...) et en particulier un précurseur reconnu et renommé dans l'étude des fossiles du bassin d'Aquitaine ${ }^{11}$. Il eut le souci, rare pour l'époque, de réunir méticuleusement ses trouvailles dans des meubles bien rangés, d'étiqueter chacun des échantillons (souvent 4 à 5 étiquettes pour un seul fossile), de les classer par groupes d'organismes, et surtout de les étudier et de les publier dans un grand nombre de "mémoires » et d'articles scientifiques où il dessina lui-même de nombreux fossiles landais. Il décrivit plusieurs centaines d'espèces nouvelles de mollusques et d'oursins, dont les «types » sont présents. Il eut donc un rôle fondateur et précurseur de la science en Aquitaine, et y créa pratiquement la paléontologie. Cette "collection Grateloup " comporte deux meubles de quinze tiroirs chacun et reste en grande partie à réviser ${ }^{12}$. 


\section{Figure 10}
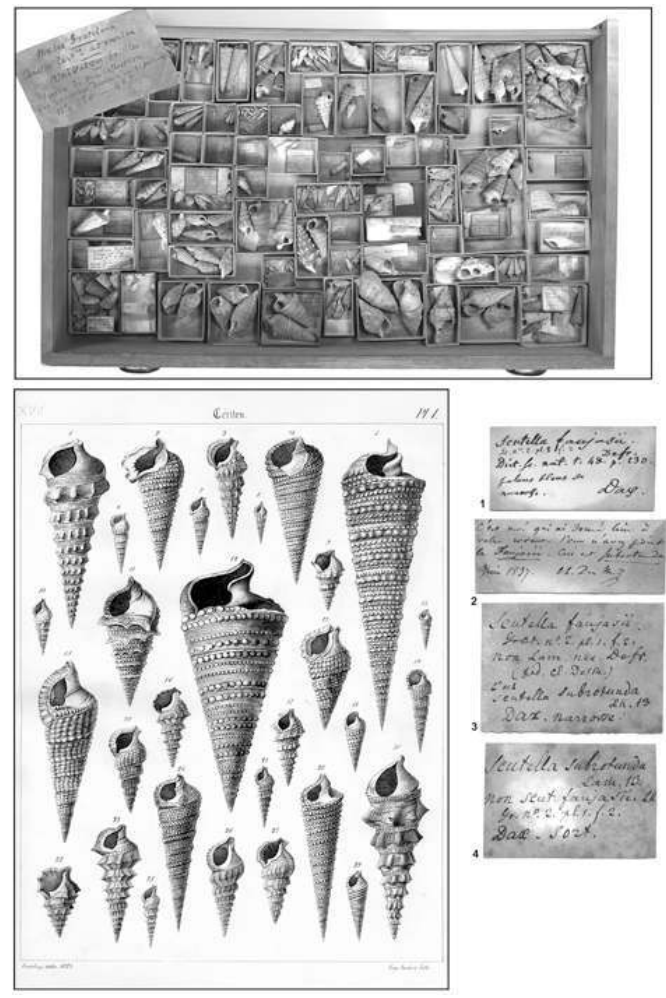

L'un des 30 tiroirs, de $65 \mathrm{~cm}$ de longueur, de la collection Grateloup, contenant des « cérithidés » du Tertiaire (gastéropodes). L'étiquette générale mentionne « Musée Grateloup. Bassin tertiaire adourien. Univalves fossiles ». En bas à gauche, exemple de planche (hauteur : $23 \mathrm{~cm}$ ) dessinée et publiée par Grateloup (1847), illustrant les « cérithes »; la plupart de ces spécimens sont des « types ou figurés ». A droite : étiquettes originelles écrites par Grateloup pour une scutelle (oursin miocène) ; la chronologie de réalisation de ces étiquettes est selon l'ordre 1, 2, 3, 4. Collections de paléontologie. Phot. Bruno Cahuzac. (C) Université Bordeaux 1. 
Figure 11

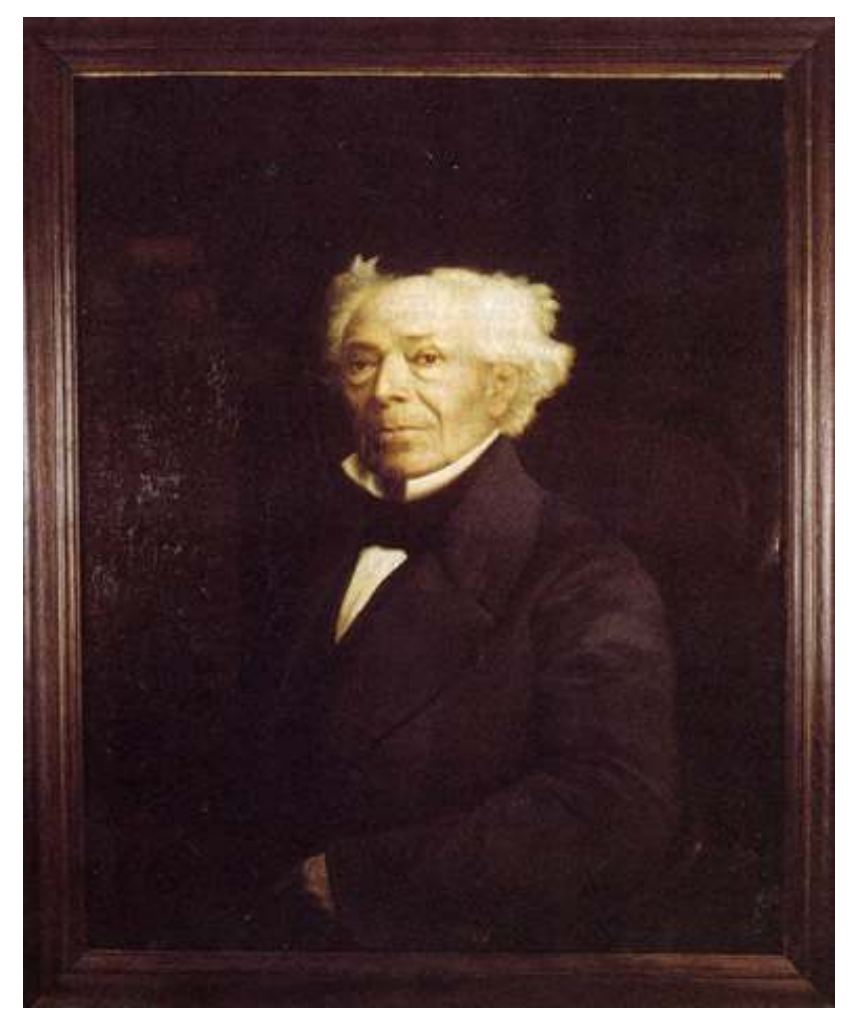

Portrait du Dr Sylvestre de Grateloup (1782-1861).

Phot. Musée de Borda à Dax. (c) Musée de Borda, Dax. Voir note $n^{\circ} 10$.

De très nombreux mollusques de l'ère tertiaire ont été décrits par la suite et figurés dans une grande publication, la «Conchologie Néogénique de l'Aquitaine », par M. Cossmann et A. Peyrot, de 1909 à $1935^{13}$. L'ouvrage entier compte des milliers de pages et plus de 120 planches. C'est "une bible" régionale encore largement utilisée aujourd'hui. Un grand nombre des spécimens types ou figurés dans cet ouvrage sont conservés dans notre université, notamment au sein de la très riche collection de M. Neuville (bien que plusieurs tiroirs de sa collection soient actuellement entreposés à l'université Pierre et Marie Curie (Paris 6) : fut-ce un prêt à Cossmann, habitant alors Paris? un don? une vente postérieure? D'autres fossiles recueillis par Neuville - des coraux surtout - sont intégrés dans la typothèque du Muséum national d'Histoire naturelle).

Parmi la microfaune, les foraminifères benthiques qui vivent sur les fonds marins constituent un groupe d'une extrême diversité morphologique et d'ornementation, et d'un grand intérêt géologique; en particulier, ils permettent de dater avec précision les dépôts marins (voir fig. ${ }^{\circ} 12$ ). Ce sont des protozoaires avec une seule cellule, de taille millimétrique à centimétrique; dans certains niveaux, on compte jusqu'à 390 espèces différentes, par exemple dans le Burdigalien supérieur marin des Landes (-17 millions d'années), récemment révisé, ce qui a permis d'enrichir la typothèque de plusieurs fossiles figurés.

Tous ces spécimens font aujourd'hui encore l'objet d'études paléontologiques diversifiées et approfondies de la part de spécialistes français et étrangers. Certains proviennent de gisements complètement disparus. De nombreux fossiles (anciennement ou nouvellement récoltés) restent encore à étudier et beaucoup d'autres devront être ré-identifiés grâce 
aux méthodes modernes d'investigation. Ces collections sont une référence irremplaçable sur le plan scientifique international, notamment grâce aux très nombreux échantillons «types» d'espèces ou figurés (dessinés ou photographiés) dans les publications paléontologiques. Consultables par tous les chercheurs, ils sont intégrés et inventoriés dans le programme national TYFIPAL (TYpes et FIgurés en PALéontologie) ${ }^{14}$. Leur saisie, incluant une iconographie, est en cours de réalisation et, à terme, ils seront accessibles par « Internet ».

41 L'université possède plus de 2000 types/figurés potentiels, nombre s'accroissant sans cesse. En effet, les recherches récentes des universitaires bordelais permettent d'enrichir les collections et d'y intégrer de nouveaux spécimens types. Ainsi, le corail " Cahuzacopsammia meandrinoides ", qui provient de l'oligocène de Saint-Paul-lès-Dax (Landes) et date de 24,6 millions d'années: ce fossile, décrit en 1999, constitue une nouvelle espèce, et même un nouveau genre, dont les exemplaires représentatifs sont devenus des références conservées dans la typothèque ${ }^{15}$. Comme les autres genres récifaux, il avait des exigences écologiques strictes et c'est donc un excellent marqueur des conditions du milieu de vie.

Les collections paléontologiques rassemblent à la fois des macrofossiles et des microfossiles (fig. $\mathbf{n}^{\circ} \mathbf{1 2}$ ) concernant principalement les ères tertiaire et secondaire (100 000 spécimens). Géographiquement, c'est le bassin d'Aquitaine qui est le mieux représenté, avec des séries complètes de tous les groupes fauniques, mais des collections générales existent aussi, utiles pour les comparaisons et pour l'aspect pédagogique. Ces fossiles permettent de dater les couches de terrain, de reconstituer les environnements successifs, les changements climatiques, les avancées des mers, et donc de connaître l'histoire de la Terre. 


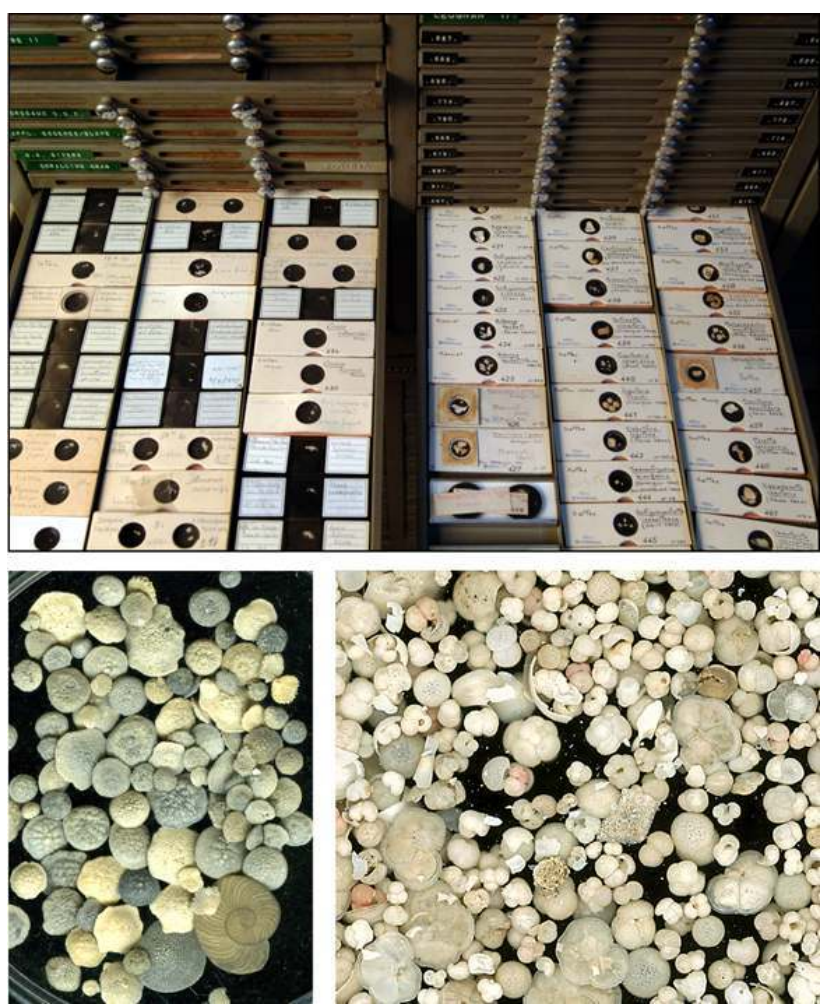

La collection de microfossiles. En bas à gauche : vue de détail de foraminifères benthiques (Oligocène supérieur des Landes; chaque coquille mesure entre 0,5 et $1 \mathrm{~cm}$ ). En bas à droite : foraminifères planctoniques (Quaternaire ; diamètre d'un test : environ 200 microns). Collections de paléontologie. Phot. Bruno Cahuzac. (C) Université Bordeaux 1.

Actuellement, ces collections sont sous la responsabilité de Bruno Cahuzac, de l'UFR des Sciences de la Terre et de la Mer.

\section{La collection carothèque océanique}

Le Département de Géologie et Océanographie déploie depuis de nombreuses années un large effort d'acquisition et de valorisation d'archives sédimentaires marines à travers les moyens mis en œuvre en mer par les instances nationales et internationales et les travaux conduits au sein de l'UMR «Environnements Paléocéanographiques et Côtiers » (EPOC). Les premières carottes conservées par l'ancien Département datent de l'époque du début de la grande aventure océanique initiée à la fin des années 1960 par le professeur Michel Vigneaux, directeur de l'Institut de Géologie du Bassin d'Aquitaine. Ces premières carottes sont déjà totalement épuisées ou inexploitables faute d'une bonne conservation. Les premiers travaux correspondent à la période de direction du Département du professeur Jean Moyes et depuis lors sont supervisés par Jean-Louis Turon.

L'organisation de nouvelles missions hauturières et la nécessité d'optimiser la conservation de ces archives ont conduit à l'équipement d'une première carothèque réfrigérée à $4^{\circ} \mathrm{C}$ en 1990 . Dès 2000, le doublement de la surface nécessaire a conduit à l'aménagement d'une seconde carothèque. L'ensemble (salles Pétrel et Albatros) 
représente une surface totale de plus de $90 \mathrm{~m}^{2}$, aujourd'hui largement saturés. Faute de place, une partie des sédiments à l'étude par les membres du laboratoire est conservée dans des grottes de la région parisienne à Gif-sur-Yvette. Le matériel ramené des campagnes océanographiques constitue une collection d'archives sédimentaires dont la finalité, à vocation essentiellement paléoclimatique, est de regrouper, conserver, gérer et fournir des sédiments aux différents demandeurs (audience internationale importante). Cette collection est éminemment vivante et continue d'accueillir régulièrement de nouveaux et nombreux tronçons de carottes sédimentaires, puisque le matériel archivé sert de support à la compréhension des changements climatiques globaux.

Anticiper le devenir du climat terrestre est un enjeu sociétal qui passe par la compréhension des mécanismes hydrologiques, atmosphériques, enregistrés notamment dans les sédiments marins. La fiabilité des prévisions climatiques à plus ou moins long terme passe, de façon incontournable, par cette compréhension. En fait, ces carottes contiennent les clés pour la modélisation du climat futur (fig. $\mathbf{n}^{\circ} \mathbf{1 3}$ ).

Figure 13

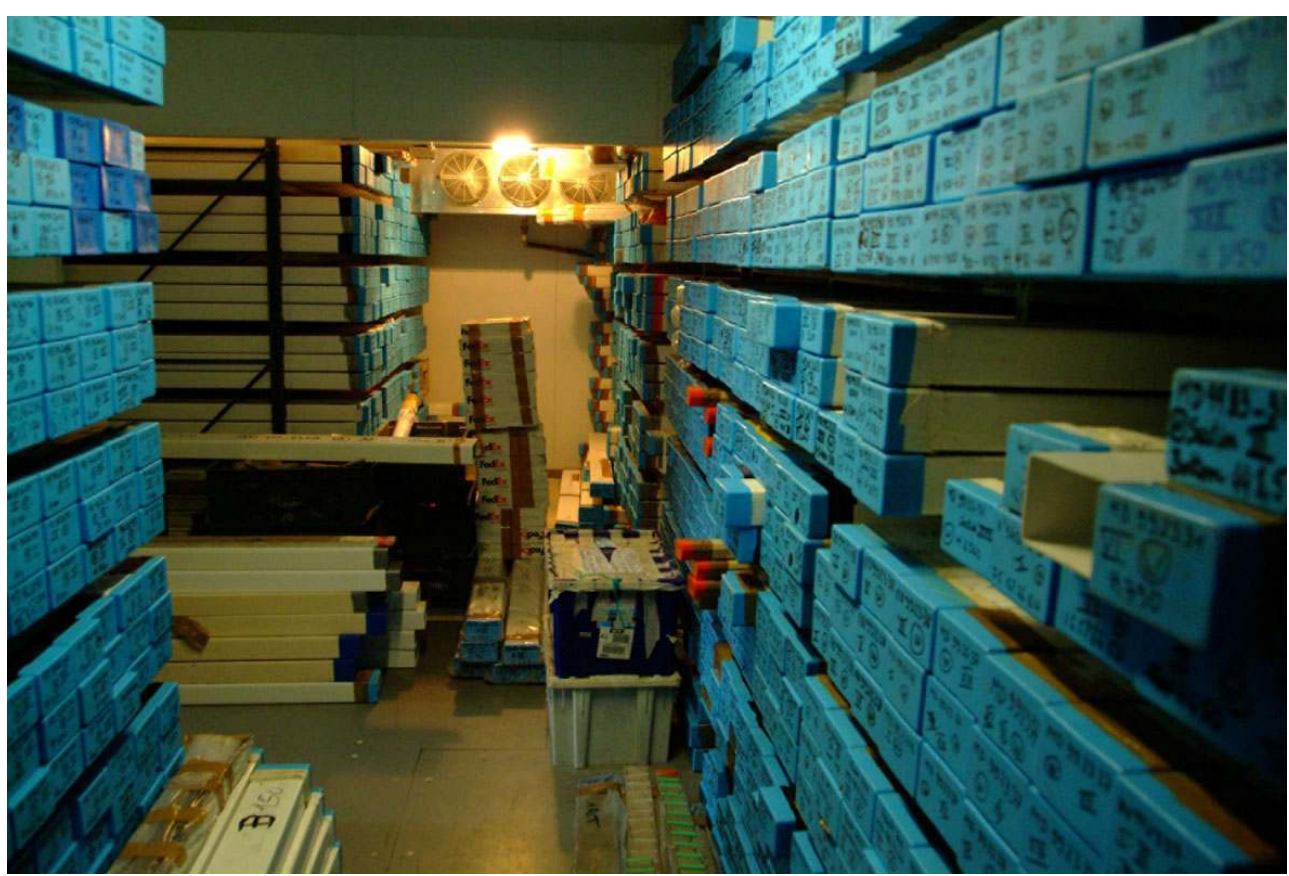

Carothèque réfrigérée des archives climatiques de l'océan. Collections du laboratoire EPOC de l'Observatoire aquitain des Sciences de l'Univers.

Phot. Pierre Baudier. @ Pierre Baudier.

À l'heure actuelle, le développement de missions océanographiques apporte annuellement à la carothèque plus de 500 mètres linéaires de sédiments, conservés et mis à la disposition de la communauté scientifique. Cette carothèque réfrigérée à $4^{\circ} \mathrm{C}$ permet une bonne conservation des sédiments, évitant ainsi une pollution bactérienne ou fongique et leur dessèchement. À ce jour, environ 2600 mètres de sédiments correspondant à plus de 150 prélèvements effectués dans tous les océans du globe (Atlantique, Méditerranée, Indien, Pacifique, Arctique et Antarctique) sont répertoriés, informatisés et conservés. Ces carottes sont des cylindres de sédiments, récupérés à des profondeurs d'eau allant de 500 à $4500 \mathrm{~m}$. 

Zoologie de la faculté des Sciences de Bordeaux. Elles se sont enrichies des collectes organisées sur le terrain par les étudiants et leurs professeurs et par l'achat de nombreux spécimens au titre de matériel pédagogique. Sujettes à diverses vicissitudes pendant la Seconde Guerre mondiale, les collections rescapées sont regroupées en 1967. Les plus belles pièces proviennent de dons de particuliers, notamment les collections de coléoptères et de papillons provenant du monde entier, rassemblées par Charles Paul Bial de Bellerade (XIX ${ }^{e}$ siècle), Émile Schirber et De Sandt (entre 1920 et 1926), ou Léopold Fournes (entre 1974 et 1977).

51 l'inventaire des muséums de France du ministère de l'Éducation nationale et sur le site de l'Office de coopération et d'information muséographiques (OCIM). Elles sont accessibles à tous par simple demande écrite auprès du responsable. La plus grande partie est exposée dans la salle des collections de biologie animale ${ }^{16}$.

Charles Paul Bial de Bellerade (1845-1918) (fig. n¹4), lieutenant d'artillerie à Besançon, blessé à Villersexel pendant la campagne de 1870-1871, entre à la compagnie des chemins de fer du Midi où il exerce les fonctions de chef du service des litiges. Passionné, depuis son enfance, par l'entomologie et l'histoire naturelle, il se spécialise au cours du temps dans l'étude des coléoptères. Il utilise ses heures de temps libre à parcourir la campagne à la recherche d'insectes et à étudier leurs mœurs et comportements. D'abord en contact avec un cercle d'amis naturalistes, il devient membre de la société entomologique de la Gironde et du Sud-ouest de la France dont il occupe les fonctions de trésorier puis de président. Plus tard, il crée un nouvel acta entomologique, « Le Zoologiste ». Entre-temps, il entre à la célèbre Société linnéenne de Bordeaux. M. Bial de Bellerade publie de nombreux travaux dans les «Actes » de la Société linnéenne, et initie même, avec MM. Coutures et Blondel de Joigny, une "Contribution à la faune des Coléoptères de la Gironde ", qui malheureusement reste inachevée. Aujourd'hui, cette contribution est toujours très moderne puisque des entomologistes de la Société linnéenne reprennent pour leurs publications les informations fournies à l'époque. En 1902, M. Bial de Bellerade 
a déjà saisi le service pédagogique que peut rendre, à la société rurale en particulier, la vulgarisation de la zoologie agricole dont il est un des premiers et des plus fervents adeptes. Ainsi, il assure une aide matérielle (par sa collection notamment) et scientifique des plus précieuses au laboratoire de la Société d'étude et de vulgarisation de la zoologie agricole. En 1914, après en avoir été membre du conseil d'administration, fonction qu'il occupe pendant plusieurs années tout en consacrant une grande partie de ses loisirs à ranger, soigner et préparer les collections élémentaires destinées aux classes rurales, il en devient finalement le vice-président. Depuis quelques années déjà, sa curiosité insatiable de naturaliste l'a fait embrasser une nouvelle passion, la conchyliologie fossile, qui bientôt n'aura plus de secrets pour lui.

Figure 14

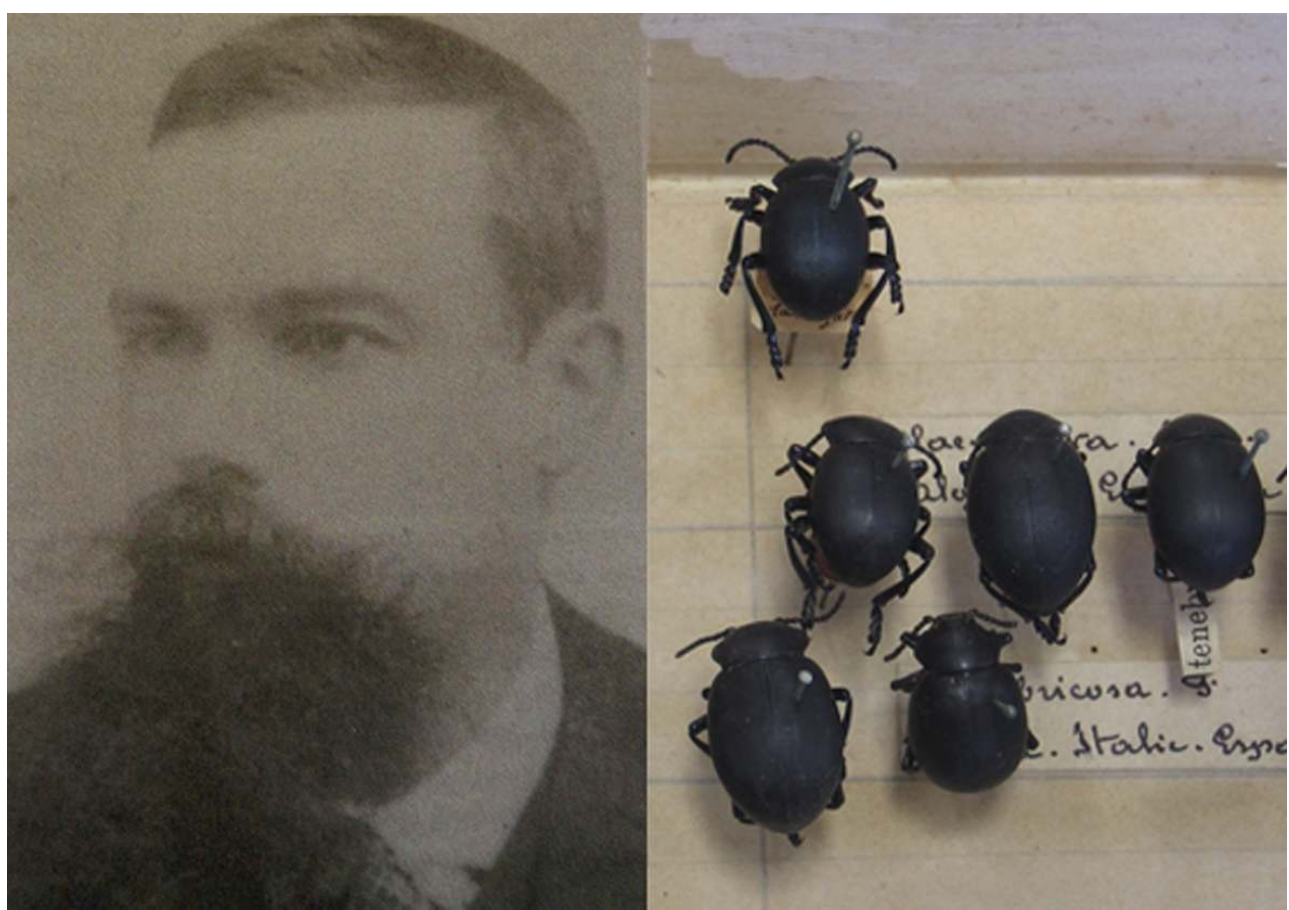

Portrait de Charles Paul Bial de Bellerade (1845-1918) associé à quelques coléoptères de la famille Timarchidae.

Phot. collections de l'UFR des Sciences biologiques. (C) Université Bordeaux 1

À son décès, il lègue à tous les entomologistes et naturalistes des générations futures des écrits précieux et une riche collection de coléoptères dont une partie est aujourd'hui conservée au Musée de biologie animale de l'université Bordeaux 1. Cette collection de coléoptères est constituée de plus de 150 boîtes renfermant près de 10000 spécimens représentatifs des populations passées et actuelles de la Gironde mais aussi des biotopes des pourtours méditerranéens (Sicile, Italie, Corse, France, Algérie, Espagne, Malte...). L'intérêt historique de cette collection vaut pour la qualité et la richesse de l'échantillonnage et témoigne d'un savoir-faire particulier dans la naturalisation et la constitution de boîtes spéciales en bois. Le choix des étiquettes et la calligraphie à l'encre de chine font de cette collection un repère temporel sur les us et pratiques du début du $\mathrm{XX}^{\mathrm{e}}$ siècle. Sa valeur scientifique est encore d'actualité puisqu'elle suscite des collaborations pour des publications scientifiques modernes : deux exemples très récents avec d'une part, la consultation de la collection par un archéoentomologiste du 
laboratoire PACEA et, d'autre part, la mise en place d'une collaboration avec un entomologiste de la Société linnéenne pour travailler sur la « contribution à la faune des coléoptères de la Gironde ».

Léopold Fournes (1942-) est sensibilisé à l'entomologie lors de ses études à Toulouse (fig. $n$ $\left.{ }^{\circ} 15\right)$. Ses études de chimie à l'université de Toulouse le mènent au doctorat de spécialité Chimie-Physique. Nommé maître-assistant au département de Chimie de l'université de Yaoundé au Cameroun en 1969, il y restera jusqu'en 1977. Au cours de ce séjour camerounais, il obtient un doctorat d'État en chimie du solide. Durant cette période, il se lie avec deux zoologistes en poste, fait avec eux ses premiers pas en entomologie et profite des connaissances et de l'expertise du professeur J.-L. Amiet avec lequel il fera de nombreuses sorties en brousse. Il constitue alors une collection de papillons du Cameroun.

Figure 15
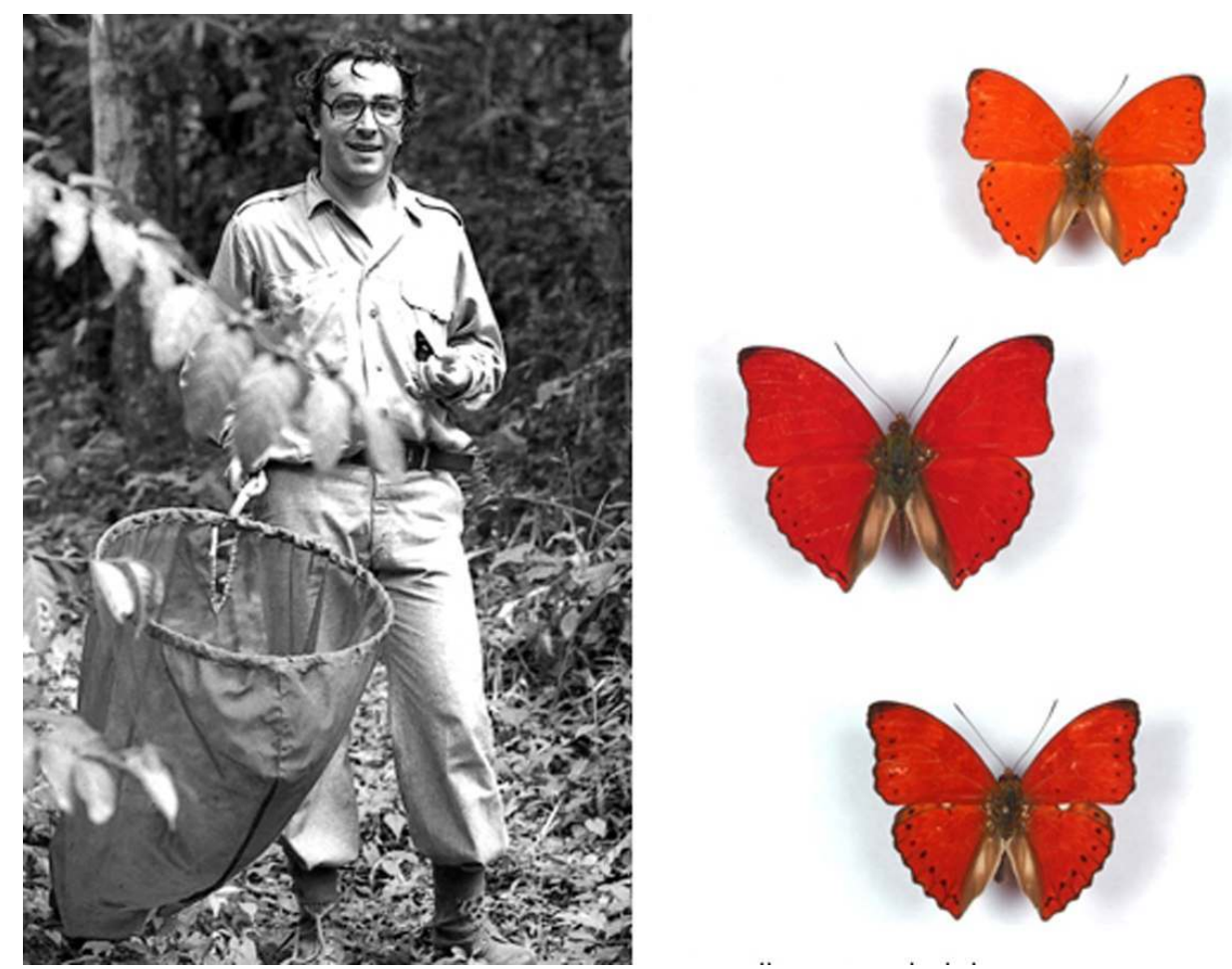

Portrait de Léopold Fournes au Cameroun en 1976, associé à quelques exemplaires de Nymphalidae du genre Cimothoe.

Phot. Collections de l'UFR des Sciences biologiques. (c) Université Bordeaux 1.

À son retour en France en 1977, L. Fournes est affecté au laboratoire de Chimie du solide URA CNRS/Bordeaux 1 où il dirige à partir de 1982 le service de spectroscopie Mössbauer. Dès lors, l'objectif principal de ses travaux est d'étudier les caractéristiques physicochimiques et structurales des matériaux solides. Pendant cette période, la collection de papillons est seulement maintenue dans un bon état de conservation.

Avec sa mise à la retraite, L. Fournes peut revenir à sa passion de l'entomologie. Il fait don, en février 2008, de sa collection au Musée de Biologie animale de Bordeaux 1 où elle est aujourd'hui conservée. Cette collection est constituée de 50 boîtes renfermant 1300 spécimens représentatifs de quatre familles (Papilionidae, Nymphalidae, Pieridae, Acraeidae) et 450 espèces différentes. La récolte de ces papillons a été effectuée sur une 
trentaine de sites localisés dans le sud-est du Cameroun de 1975 à 1977. Ce biotope appartient à la zone forestière afro-tropicale. Cette collection est intéressante car les collectes ont été effectuées sur une zone géographique relativement réduite et sur un biotope très homogène. Tous les échantillons sont en bon état de conservation et systématiquement répertoriés, ce qui peut être utile à une étude de la biodiversité. En outre, la richesse en spécimens de certains genres comme cymothoe (famille des Nymphalidae) peut permettre une étude poussée sur le polymorphisme sexuel de ces animaux.

Cette collection complète parfaitement les deux autres collections de papillons déjà conservées au Musée de Biologie animale: d'une part, une collection "papillons du monde » réalisée au tout début $\mathrm{du} \mathrm{XX}^{\mathrm{e}}$ siècle et présentant des papillons des quatre coins du globe, d'autre part, une collection " papillons de nos contrées » réalisée au long du XX siècle et présentant des papillons de France.

58 Actuellement, cette collection est sous la responsabilité de Jean-Rémi Pape et Andrée Boutin, de l'UFR de Sciences Biologiques.

\section{La collection d'Astronomie}

La collection de bâtiments, d'instruments d'observation et d'instruments divers, de clichés, d'archives et d'ouvrages d'astronomie de l'université Bordeaux 1 a été constituée dès la fondation de l'observatoire astronomique de Bordeaux à Floirac en $1877^{17}$. Quelques petits instruments et des ouvrages anciens viennent de l'ancien observatoire de l'Académie des Sciences, Belles-Lettres et Arts de Bordeaux et de la chaire d'astronomie et mécanique rationnelle, l'une des six chaires fondées lors de la création de la faculté des Sciences de Bordeaux par ordonnance royale du 24 août 1838. Enfin, cette collection a été complétée au fil des décennies en fonction de l'évolution des thématiques scientifiques traitées jusqu'à nos jours : météorologie, astrométrie, en particulier la radioastronomie à partir des années 1960, puis l'aéronomie et la planétologie. Cette collection regroupe des bâtiments, de grands instruments, une large collection d'instruments divers, une collection de clichés d'astrométrie, des ouvrages et revues, des dossiers d'archives, des objets divers comme aquarelles d'observation, du mobilier, etc.

Les instruments d'observation sont contenus dans des bâtiments spécifiques. Trois bâtiments contenant respectivement une lunette méridienne (fig. $\mathbf{n}^{\circ} \mathbf{1 6}$ ), une grande lunette équatoriale et une petite lunette équatoriale, furent installés de 1879 à 1882 , et celui de l'équatorial photographique en 1890 sous la direction de Georges Rayet (1839-1906). Le bâtiment principal et celui de la table équatoriale furent construits de 1940 à 1942 sous la direction de Gilbert Rougier (1886-1947). Un bâtiment de bureaux fut construit en 1968 par Pierre Sémirot (1907-1972) et complété par une nouvelle aile en 1986 réalisée par Jérôme de La Noë (1941-). 


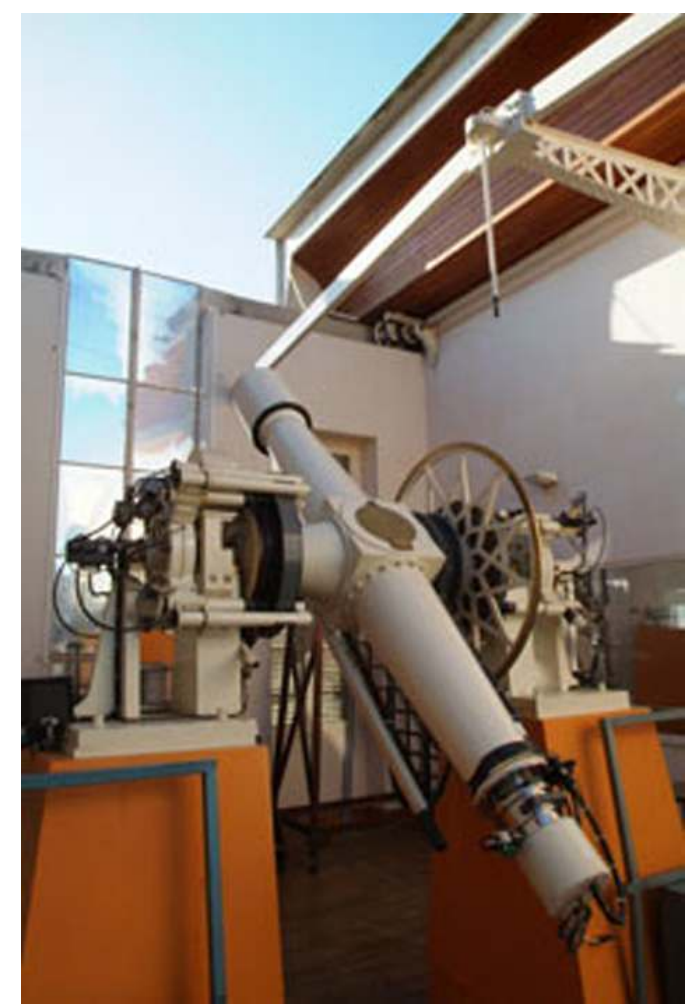

Lunette méridienne de l'observatoire astronomique de Bordeaux à Floirac. Installée en 1882, rénovée et contrôlée par ordinateur dans les années 1980, elle est toujours opérationnelle et utilisée pour des mesures de positions d'astres. Collections Observatoire Aquitain des Sciences de l'Univers.

Phot. OASU/LAB. @ Université Bordeaux 1.

61 Un radiotélescope fut installé en 1965, utilisant un miroir parabolique fabriqué à Würzburg (ancien radar allemand de la côte normande) par Pierre Sémirot, et un interféromètre de deux radiotélescopes de 2,50 $\mathrm{m}$ de diamètre fut installé en 1972 par Jean Delannoy (1931-) afin de préfigurer l'interféromètre à 6 antennes en ondes millimétriques du Plateau de Bure de l'Institut de Radio-Astronomie Millimétrique (IRAM), institut franco-allemand-espagnol.

62 La collection de théodolites, et de petits instruments d'horlogerie, de spectroscopie, de photographie, etc., a été constituée au fil des décennies pour contribuer à la réalisation des projets scientifiques (fig. $\left.\mathbf{n}^{\circ} \mathbf{1 7}\right)$. De même la collection d'ouvrages d'astronomie, de mathématiques appliquées et d'astrophysique a été augmentée régulièrement par l'acquisition d'ouvrages et de revues du XIX ${ }^{\mathrm{e}}$ siècle à nos jours, pour les besoins des astronomes. 


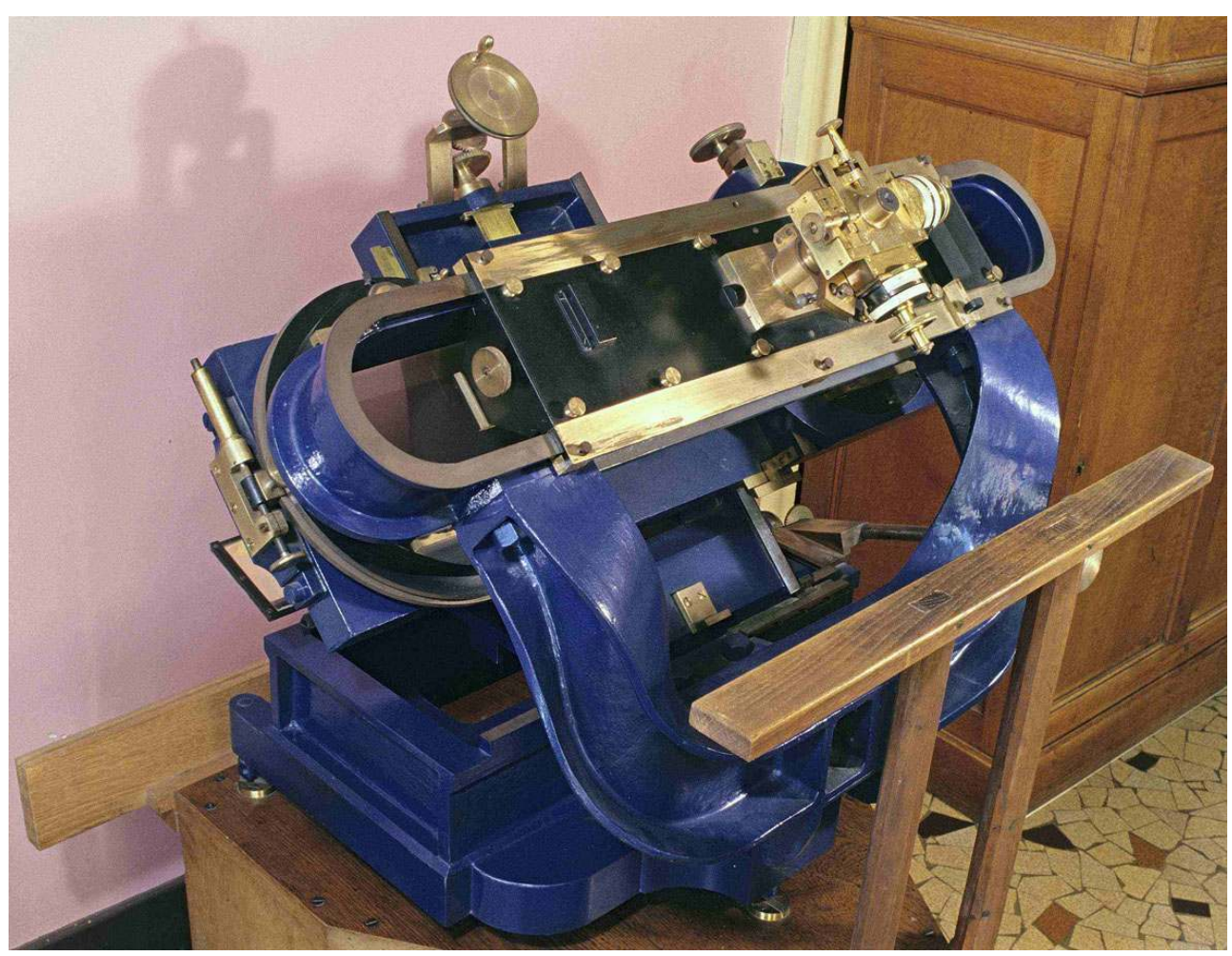

Stéréo comparateur fabriqué par Prin, Paris, en 1910. Cette machine a pour but de détecter rapidement sur des plaques photographiques des astres à grand déplacement (planètes, astéroïdes, comètes...) ou des astres dont l'éclat varie (novae, supernovae, étoiles variables).

Phot. Michel Dubau. @ Région Aquitaine, Inventaire général, 2004.

\section{laboratoire d'électronique, un atelier de mécanique, un service informatique, et la salle de} la lunette méridienne, trois coupoles comportant une grande et une petite lunette équatoriale, une lunette équatoriale photographique pour la «Carte du ciel $»^{18}$, une coupole pour la table équatoriale et son télescope de $60 \mathrm{~cm}$ de diamètre, un bâtiment pour des mesures de courants telluriques et de champ magnétique, la maison de maitre qui sert de logement au directeur, cinq maisons d'astronomes, une maison réservée aux visiteurs de passage et la conciergerie.

Les instruments sont une lunette méridienne (1880), deux lunettes équatoriales (1882), une lunette équatoriale photographique (1890), un radiotélescope de Würzburg (1965) ayant servi à la mesure du flux solaire, une des deux antennes de 2,50 $\mathrm{m}$ de diamètre de l'interféromètre (1972) ayant servi à l'étude du milieu interstellaire. Un radiomètre micro-onde a été construit et utilisé pour l'étude de la couche d'ozone stratosphérique. L'observatoire dispose également d'un certain nombre de petits instruments, plus de 500 environ, utilisés pour diverses applications en astronomie: horloges, baromètres, chronographes, spectrographes, viseurs, appareils photographiques, etc. Un premier inventaire a été effectué en 1984 et repris et complété en 2004 lors d'un inventaire exhaustif effectué dans le cadre d'une convention entre le ministère de la Recherche et le ministère de la Culture qui a pris en charge une campagne photographique réalisée par le photographe de la DRAC Aquitaine. 

position actuelle d'étoiles mesurée sur des clichés plus récents obtenus par l'équatorial photographique, avec celle mesurée sur des clichés anciens de plus de cent ans, il est possible de déterminer leur mouvement propre. Enfin, une cinquantaine de clichés sur plaques de verre illustrent les débuts de l'observatoire. Près de deux cents dossiers d'archives contiennent la vie scientifique, administrative et financière de l'observatoire depuis sa fondation. S'y ajoutent diverses aquarelles réalisées au cours de l'observation de comètes ou planètes, du mobilier de bureau, table et fauteuils fin XIX ${ }^{\mathrm{e}}$, quelques meubles spécifiques: servantes, supports, etc. La collection comporte environ un millier d'ouvrages d'astronomie, d'astrophysique et de disciplines associées dont 250 des XVIII ${ }^{e}$ et XIXe siècles. À ces ouvrages en cours d'inventaire, il faut ajouter les collections de journaux et revues spécifiques à l'astronomie en général.

66 L'intérêt de cette collection réside dans le fait qu'elle a été constituée au fil des jours sur plus d'un siècle pour les besoins de la recherche et de l'enseignement en astronomie et astrophysique. L'ensemble de la collection retrace la vie passée de l'établissement, les projets scientifiques, les mesures au jour le jour, les calculs, la publication des résultats, l'évolution des technologies et des techniques utilisées souvent en avant-garde, les collaborations locales, nationales et internationales.

67 Au-delà de leur intérêt purement scientifique et technique ainsi qu'esthétique, les instruments d'astronomie de l'observatoire de Bordeaux contribuent à l'étude de l'histoire de l'instrumentation et de la méthodologie de la démarche scientifique depuis près de cent-trente ans. La collection présente donc un grand intérêt en histoire des sciences et en pédagogie vis-à-vis des jeunes générations. C'est l'objet du projet actuel de conservation et de développement vers le public, qui verrait le jour lorsque les équipes $\mathrm{du}$ Laboratoire d'Astrophysique de Bordeaux auront été transférées sur le campus de Talence.

68 Actuellement, cette collection est sous la responsabilité de Jérôme de La Noë et JeanMichel Rousseau, du Laboratoire d'Astrophysique de Bordeaux.

\section{Les collections de la Bibliothèque universitaire des sciences et technologies}

Le Service commun de la Documentation (SCD) de l'université Bordeaux 1 est chargé de mettre en œuvre la politique documentaire de l'université, dont l'objectif est d'assurer à la communauté scientifique universitaire - étudiants comme enseignants-chercheurs l'accès à toute la documentation requise par l'enseignement et la recherche dans les domaines couverts par l'université. Les quatre principales fonctions relevant de cette mission sont d'abord l'acquisition, le traitement et la communication de la documentation scientifique, puis la formation des utilisateurs à la recherche documentaire et à l'usage de la documentation, et notamment aux techniques les plus récentes d'accès à cette documentation. Ensuite, on trouve la participation à la production et à la diffusion de l'information scientifique, ainsi qu'aux activités d'animation culturelle, scientifique et technique de l'université, et enfin la coopération avec d'autres bibliothèques ou organismes documentaires, qui concourent aux mêmes objectifs. Toutes ces missions s'articulent autour des collections et ressources mises à disposition par le SCD. 
70 Au sein du SCD de Bordeaux 1, la Bibliothèque universitaire des Sciences et Techniques (BUST) offre à la consultation 102000 livres, 17000 thèses, 2152 titres de périodiques sous forme papier dont 280 en cours d'abonnement, ainsi que de la documentation sur d'autres supports (microformes, cédéroms, vidéos...). Les lecteurs ont également accès sur place ou à distance aux 5600 périodiques, ouvrages et bases de données électroniques mis à disposition par le Service commun de la documentation.

71 Parmi ses collections, la BUST compte des fonds patrimoniaux constitués d'ouvrages acquis depuis la création de la bibliothèque en 1838 jusqu'en 1914, évalués à 35000 volumes (livres, périodiques et thèses) dont 1531 ouvrages antérieurs à 1811. Les fonds sont encyclopédiques dans les domaines scientifiques et techniques. Acquis pour l'usage immédiat des universitaires, ils sont essentiellement composés de documents du $\mathrm{XIX}^{\mathrm{e}}$ siècle avec quelques points forts en chimie, mathématiques, paléontologie, botanique et astronomie, auxquels s'ajoute une collection de livres anciens particulièrement intéressants en mathématiques, chimie, alchimie et astronomie. La bibliothèque conserve des textes importants à l'origine de la science. L'un des ouvrages les plus précieux est le "De revolutionus orbium caeslestium» de Copernic qui a inauguré l'héliocentrisme et donné une nouvelle vision de l'univers. Quelques incunables, une provenance illustre - celle de 80 ouvrages de la bibliothèque de Lavoisier avec son exlibris (fig. $\left.\mathbf{n}^{\mathbf{0} 18}\right)^{19}$-, de belles reliures, ainsi que de belles illustrations de botanique ou de zoologie: les gravures sur bois des ouvrages de Gesner ou Aldrovandi, les oiseaux en grand format de Buffon, de délicates lithographies des oiseaux d'Amérique d'Audubon, constituent l'essentiel des richesses bibliophiliques du fonds ancien ${ }^{20}$. Les conditions de sécurité et de conservation sont globalement bonnes, mais la place dans les réserves commence à manquer. 


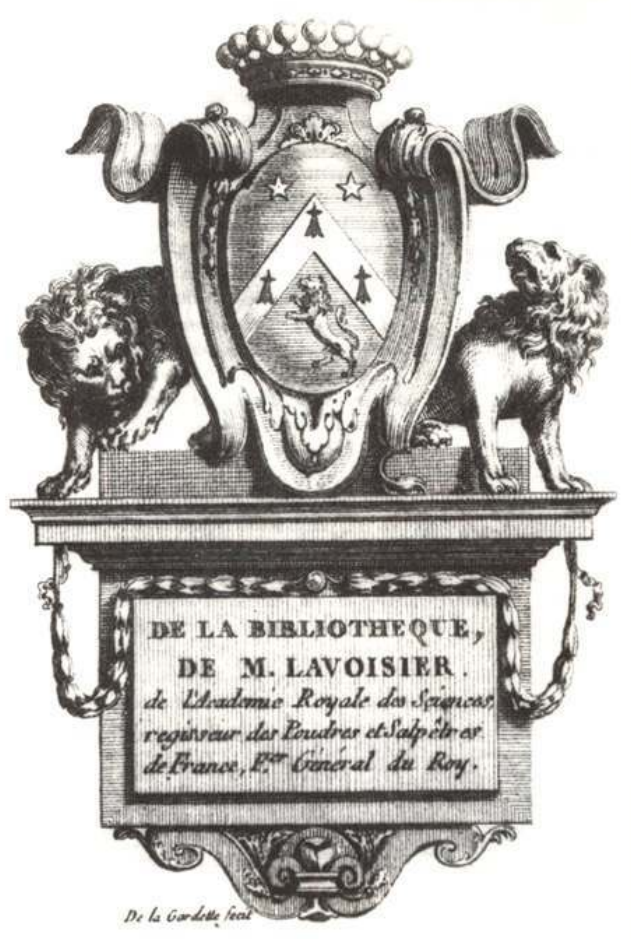

Ex-libris gravé de Lavoisier. Collections de la Bibliothèque universitaire des Sciences et Techniques, Université Bordeaux 1.

Phot. BUST. @ Université Bordeaux 1.

Les ouvrages sont entrés dans les collections de la bibliothèque essentiellement par achat, mais aussi par échange pour les thèses, et par don, notamment du ministère de l'Instruction publique. Ce dernier prenait en charge l'édition et la diffusion des résultats de missions scientifiques, ou d'œuvres d'auteurs scientifiques importants comme Lavoisier ou Fermat. En 1838, à la création de la faculté des Sciences et de la faculté des Lettres de Bordeaux, une bibliothèque commune est prévue avec une somme de 2000 francs allouée pour l'achat de livres scientifiques. En réalité, la bibliothèque n'existe que de nom pendant longtemps, mais malgré tout elle s'accroît, lentement jusqu'en 1870, puis plus rapidement. En 1879, une salle spéciale lui est attribuée et elle devient universitaire par attribution d'un budget propre et de personnel professionnel. En 1895, les facultés des Sciences et des Lettres s'installent dans des locaux plus vastes, dans le Palais des facultés, cours Pasteur et la bibliothèque est dotée d'un local adapté, qu'elle ne quitte qu'en 1965 pour les locaux du campus de Talence. Les bibliothèques des lettres et des sciences vont alors chacune dans un bâtiment séparé21.

Un enrichissement sensible en livres anciens a lieu à la fin du XIX et au début du XX ${ }^{e}$ siècle. Il est le résultat de legs ou d'achats d'ouvrages ayant appartenu à des universitaires bordelais : Alexandre Baudrimont (1806-1880), professeur de chimie ; Jules Hoüel (1823-1886), professeur de mathématiques; Gaston Lespiault (1823-1904), professeur de mécanique. Au XXe siècle, d'autres dons se succèdent: Pierre Duhem (1861-1916), professeur de physique ; Léonce Motelay (1830-1917), botaniste, puis Édouard Harlé (1850-1922), ingénieur des Ponts et Chaussées et préhistorien, et les collections de 
la Société des Sciences physiques et naturelles. Le plus récent don d'importance est celui de la bibliothèque scientifique personnelle d'Adolphe Pacault, effectué par son fils au décès de son père en 2008, suivi en 2009 par celui du Centre de Recherche Paul Pascal, concernant la bibliothèque de travail de cet illustre chercheur. Ces ouvrages, essentiellement du XXe siècle, rejoindront les collections récentes de la bibliothèque, mais leur provenance sera stipulée au catalogue afin de permettre des recherches futures. Les collections anciennes servent encore de nos jours l'enseignement et la recherche. L'enseignement des différentes disciplines scientifiques nécessite l'éclairage de l'histoire des sciences, la découverte et la connaissance des textes fondateurs. De même, dans de nombreux domaines, les recherches actuelles se nourrissent de travaux publiés dans les siècles passés, réexaminés et réinterprétés à l'aune des savoirs les plus récents. Les lecteurs peuvent examiner sur place les ouvrages originaux conservés à la bibliothèque ou bien consulter des fac-similés sous forme imprimée ou numérique, ainsi que des éditions critiques. Différentes données historiques et bibliographiques, procurées notamment par l'étude des catalogues et inventaires des bibliothèques et des archives, permettent d'enrichir la connaissance de l'histoire des textes. Un fonds d'ouvrages récents sur l'histoire des sciences et l'épistémologie, des biographies de savants et un fonds d'usuels de référence par discipline sont également à disposition des lecteurs.

D'usage immédiat, les acquisitions actuelles du SCD constituent aussi les collections patrimoniales de demain. Or, plus des deux tiers du budget d'acquisition du SCD sont consacrés actuellement à l'acquisition de la documentation électronique disponible en ligne en bibliothèque, dans les laboratoires ou par profil itinérant à partir du site de l'université : 5600 revues électroniques, 14 bases de données, 10 encyclopédies ainsi que des manuels de laboratoires, des livres numériques (2 685 actuellement, 6540 environ d'ici fin 2009). Les abonnements aux revues électroniques permettent la consultation d'archives, de plus en plus anciennes au fil des ans et des négociations commerciales avec les éditeurs. Bientôt, les bibliothèques offriront principalement des accès virtuels à des documents électroniques conservés ailleurs. La collection, dématérialisée, sera constituée de sommes d'accès. Il s'agit d'un changement radical en termes d'offre (démultipliée) et d'usage (facilitation des possibilités de recherche, consultation à domicile ou dans les laboratoires, etc.). En termes de conservation, les difficultés demeurent, remplacées par des contraintes techniques différentes, liées au support et à l'économie de l'édition électronique. Cependant, dans un tel contexte, la visibilité des ouvrages patrimoniaux passe de plus en plus par leur numérisation et leur diffusion sur Internet dans le sillage des grandes entreprises de numérisation comme Gallica, Europeana, Googlebooks, mais aussi d'autres plus modestes et très spécialisées, comme par exemple les Bibliothèques virtuelles humanistes de Tours pour les ouvrages humanistes de la Renaissance ou BibNum pour les textes scientifiques antérieurs à 1940 .

Une bibliothèque universitaire est également un lieu de travail, d'études et d'échanges qui peut se concevoir en un certain sens comme une version moderne, élargie et démocratisée, des anciens cabinets de curiosités et d'études. C'est son usage et la vie intellectuelle générée autour de ses collections qui en font véritablement la valeur. Les collections doivent être accessibles et connues. Elles ne vivent que par les étudiants et enseignants-chercheurs auxquels elles s'adressent, par les apprentissages, échanges et travaux qu'elles suscitent, par leur proximité ou leur lien avec les autres collections existantes ailleurs. Il en va de même des dons et des legs privés faits aux bibliothèques et aux archives. Ils permettent d'offrir une collection à un public plus large et averti, au sein 
d'un environnement favorable à sa consultation. C'est pourquoi la BUST présente dans ses locaux des expositions et des animations (rencontres, conférences) où les ouvrages anciens tiennent une place importante. De même, les bibliothèques numériques désormais accessibles en ligne doivent à leur tour se soucier de faire sens afin de rencontrer leur public où qu'il se trouve. Inventaires et catalogues raisonnés, bibliothèques numériques thématiques, expositions virtuelles, ces outils doivent permettre les échanges et l'interopérabilité avec les autres grands réservoirs de documents numériques et réseaux documentaires afin de constituer des puissants outils de recherche et de valorisation. Après avoir procédé l'année dernière à une grande opération de rétro-conversion de ses anciens fichiers papier désormais versés dans les catalogues informatisés SUDOC et BABORD, la BUST s'efforce d'améliorer la consultation et la valorisation de ses ouvrages anciens en s'engageant dans des opérations ponctuelles de numérisation, en collaboration avec des enseignants et des chercheurs. Les résultats, encore modestes actuellement, devraient être visibles plus largement dans les mois à venir.

77 Actuellement, la collection d'ouvrages patrimoniaux de la BUST est sous la responsabilité de Mylène Surville et Claire-Lise Gauvain.

\section{Conclusions}

78 La description des collections de l'université Bordeaux 1 qui vient d'être énoncée, révèle largement combien ces collections sont immenses, riches, et d'une extraordinaire variété. Essentiellement dévolues à l'enseignement et la recherche universitaires, elles constituent également un patrimoine considérable et passionnant. Elles ont été constituées au fil des décennies par des collectionneurs et des collecteurs aux motivations profondes allant parfois jusqu'à la passion. Si une majorité de ces collectionneurs sont des universitaires, on y rencontre également des hommes exerçant d'autres professions parfois très éloignées des objets collectionnés, tels Marcel Neuville ébéniste, E. Bonnel randonneur pyrénéiste, Sylvestre de Grateloup médecin, et bien d'autres entièrement pris par leur passion. Certes, bien des universitaires collectionneurs étaient également animés par une certaine passion mais plus axée sur la qualité de leur enseignement et de leurs recherches.

79 Les responsables actuels des collections sont aussi passionnés car, connaissant bien les collections qu'ils gèrent, ils sont conscients de leur valeur, non seulement comme témoins des travaux de leurs prédécesseurs mais surtout comme éléments fondamentaux pour l'enseignement et la recherche actuels. Ces collections participent également de l'histoire des sciences en ce sens qu'elles permettent d'appréhender les démarches scientifiques et les méthodologies utilisées par les hommes en général, les universitaires et les chercheurs.

80 Mais les responsables sont aussi conscients que ces collections demeurent peu connues et sont actuellement, pour certaines, conservées dans des conditions insatisfaisantes, mettant gravement en danger leur préservation. De plus, la plupart sont peu mises à la disposition des publics, ce qui nécessiterait un travail spécifique de mise en forme à réaliser avec des scénaristes et professionnels de l'exposition.

81 Ces raisons ne font que renforcer l'idée que l'université Bordeaux 1 a la nécessité de disposer d'un poste de "conservateur » pour gérer, conserver, réfléchir aux meilleurs 
moyens de conservation, de mise à disposition, à la fois aux usagers universitaires mais aussi aux publics, de l'ensemble des collections. En particulier, il faut absolument réunir de meilleures conditions de stockage, hors d'eau et autres nuisances, d'accessibilité et de mise en valeur.

En espérant l'affectation d'un tel poste et l'arrivée d'un personnel compétent, les responsables de collections ont lancé le projet d'exposition avec Cap Sciences pour une réalisation en 2010, et certains ont participé aux Journées du Patrimoine et à la Fête de la Science en 2009.

\section{NOTES}

1. - Co-auteurs de cet article : Dominique Armand, Univ. Bordeaux, PACEA, UMR 5199 ; Maryelle Bessou, Univ. Bordeaux, PACEA-A3P, UMR 5199, UFR de Sciences Biologiques, PACEA-LAPP; Andrée Boutin, Univ. Bordeaux, UFR de Sciences Biologiques, Centre de Neurosciences intégratives cognitives; Bruno Cahuzac, Univ. Bordeaux, UFR des Sciences de la Terre et de la Mer, Laboratoire de Recherches et Applications Géologiques; Patrice Courtaud, Univ. Bordeaux, PACEA-A3P, UMR 5199, UFR de Sciences Biologiques, PACEA-LAPP ; Claire-Lise Gauvain, Univ. Bordeaux, BUST, Bibliothèque universitaire des Sciences et Techniques, Service commun de documentation; Laurent Lachaud, Muséum d'Histoire naturelle de Bordeaux ; Laurent Londeix, Univ. Bordeaux, EPOC, UMR 5805, Observatoire aquitain des Sciences de l'univers, Environnements et Paléoenvironnements Océaniques; Nathalie Mémoire, Muséum d'Histoire naturelle de Bordeaux; Jean-Rémi Pape, Univ. Bordeaux, INCIA-UMR 5287, UFR de Sciences Biologiques, Centre de Neurosciences intégratives cognitives; Éric Pubert, Univ. Bordeaux, PACEA-PPP, UMR 5199 ; Jean-Michel Rousseau, Univ. Bordeaux, OASU/LAB, UMR 5804 ; Mylène Surville, Univ. Bordeaux, Bibliothèque universitaire des Sciences et Techniques, Service commun de documentation; Jean-Louis Turon, Univ. Bordeaux, EPOC, UMR 5805, Observatoire aquitain des Sciences de l'univers, Environnements et Paléoenvironnements Océaniques.

2. - COLLECTIF. Université Bordeaux 1 : Les collections. Talence : édition Université Bordeaux 1, 2006.

3. - COLLECTIF. Université Bordeaux 1: Vidéo sur les collections, Service Culture-Communication, Université Bordeaux 1, Réalisation « Tête à claps ", 2003-2004.

4. - http://www.pacea.u-bordeaux1.fr/presentationipgq.html

5. - GOMEZ DE SOTO, José. « Docteur Raymond Riquet (1914-1983)». Bulletins et Mémoires de la Société Archéologique et Historique de la Charente, Angoulême, 1984, n¹, p. 25-26.

6. - COURTAUD, Patrice, DUDAY, Henri. «Gestion et étude des séries anthropologiques, Centre anthropologique de Pessac (Gironde) ». Les collections ostéologiques humaines, Bulletin Archéologique de Provence, Supplément 4, 2006, p. 33-36.

7. - RAYET, Georges. «Histoire de la Faculté des Sciences de Bordeaux (1838-1894)». Actes de l'Académie des Sciences, Belles-Lettres et Arts de Bordeaux, 1897, 3e série, 59 année, p. 5-369.

8. - RAULIN, Victor. "Coupes géologiques des sondages exécutés dans le Sud-Ouest de la France (Gironde, Auch et Dax) par feu Timothée Billiot». Actes de la Société Linnéenne de Bordeaux, 1868, tome 26 (1866-1868), p. 241-268.

9. Société linnéenne de Bordeaux [Collectif]. «Fernand Daguin (1889-1948)». Actes de la Société Linnéenne de Bordeaux, 1950, tome 94 (1947 à 1950). 
10. - Pour la photo 11, il nous est demandé d'ajouter la mention suivante: «La Ville de Dax dispose des droits relatifs à la propriété, à la représentation, à la reproduction et la commercialisation de toutes les œuvres du musée de Borda. Toute violation constatée de ces droits entraînera des poursuites judiciaires ».

11. - CAHUZAC, Bruno. «Le Docteur Grateloup, naturaliste précurseur en paléontologie landaise. I - Aperçu sur sa vie et grands traits de son œuvre scientifique ». Bulletin de la Société de Borda, Dax, 2001, $126^{\mathrm{e}}$ année, 462 (3), p. 367-414.

12. - CAHUZAC, Bruno, DUPUY, Jean-Pierre, LAVERGNE, Guy. «Révision des oursins fossiles d'Aquitaine décrits par Grateloup (1836), et en partie retrouvés dans sa collection conservée à l'Université Bordeaux-1 ». Cossmanniana, Paris, 2005, tome 10 (1-4), p. 1-79.

13. - COSSMANN, Maurice, PEYROT, Albert, 1909-1923, puis PEYROT, Albert, 1925-1935. «Conchologie néogénique de l'Aquitaine ». Actes de la Société Linnéenne de Bordeaux, tomes 63-86.

14. - Inventaire national des types et figurés paléontologiques : http://transtyfipal.ubourgogne.fr.

15. - CHAIX, Christian. "Cahuzacopsammia meandrinoides nov. gen. et sp., Scléractiniaire Dendrophylliidé de l'oligocène supérieur d'Aquitaine (France) ». Geobios, Lyon, 1999, vol. 32, 6, p. 805-813.

16. - http://www.u-bordeaux1.fr/collections_biologie/.

17. - MAISON, Laetitia. La fondation et les premiers travaux de l'observatoire astronomique de Bordeaux (1871-1906): Histoire d'une réorientation scientifique. Thèse de doctorat d'épistémologie et d'histoire des sciences de l'Université Bordeaux 1, 2004, $431 \mathrm{p}$.

18. - LE GUET-TULLY, Françoise, DE LA NOË, Jérôme, SADSAOUD, Hamid. « L'opération de la Carte $\mathrm{du}$ ciel dans les contextes institutionnel et technique de l'astronomie française à la fin du XIX ${ }^{\mathrm{e}}$ siècle ». La Carte du Ciel, histoire et actualité d'un projet scientifique international, Jérôme Lamy, Paris, EDP Sciences, 2008, p. 69-107.

19. - MAURY, René. Lavoisier, ex-libris : une collection bordelaise. Bordeaux: Service interétablissement de coopération documentaire - Bibliothèque universitaire des sciences et techniques, 1995.

20. - MAURY, René. "Bibliothèque universitaire des sciences». Patrimoine des bibliothèques de France. Vol. 7 : Aquitaine, Languedoc-Roussillon, Midi-Pyrénées, Paris, Payot, 1995, p. 87.

21. - RAYET, Georges. «Histoire de la Faculté des Sciences de Bordeaux (1838-1894)». Actes de l'Académie des Sciences, Belles-Lettres et Arts de Bordeaux, 1897, 3e série, 59e année, p. 69-72.

\section{RÉSUMÉS}

De nombreuses universités scientifiques possèdent des collections. L'université Bordeaux 1 possède neuf grandes collections concernant les domaines de la préhistoire, de l'anthropologie, de la géologie terrestre et marine, de la paléontologie et micropaléontologie, de la biologie, de l'astronomie et d'un fonds ancien et moderne d'ouvrages scientifiques. Certaines collections sont riches de milliers de spécimens: fossiles d'invertébrés, ossements de vertébrés, silex taillés, ossements humains préhistoriques ou historiques, animaux naturalisés, carottes de sédiments, instruments d'astronomie et livres anciens. Ces collections ont été constituées par des privés ou des chercheurs et ont ensuite été léguées à l'université. Il est important d'identifier les collectionneurs et surtout les motivations qui ont guidé leurs choix : recherche, enseignement, 
passion. Entre histoires personnelles et histoires locales, ce regard sur les collections est aussi une porte ouverte sur l'histoire des Sciences.

Most major scientific universities own numerous collections. The Bordeaux 1 University has nine large collections in various scientific fields such as Prehistory, Anthropology, Geology, Oceanography, Palaeontology and Micropalaeontology, Biology, Astronomy and patrimonial and modern scientific books and documentation. Some collections are wealth of thousands of specimens : invertebrate fossils, vertebrate bones, flints, prehistoric and historic human bones, naturalised animals, sediment cores, astronomy instruments et old books. Such collections were built up by private persons, researchers and academics who have bequeathed them to the university. It is quite important to identify all collectors and mainly to understand what were the motives which guided their choices : research, teaching, passion. Between personal and local histories, such a look at university collections is also an open door to history of sciences.

\section{INDEX}

Mots-clés : collections universitaires, préhistoire, anthropologie, géologie, minéralogie, paléontologie, micropaléontologie, océanographie, biologie animale, astronomie, patrimoine écrit

\section{AUTEUR}

\section{JÉRÔME DE LA NOË}

Directeur de recherches CNRS honoraire, Observatoire aquitain des Sciences de l'univers, Laboratoire d'Astrophysique de Bordeaux delanoe@obs.u-bordeaux1.fr 